Article

\title{
Structure Elucidation and Toxicity Analysis of the Byproducts Formed after Biodegradation of Aflatoxins B1 and B2 Using Extracts of Mentha arvensis
}

\author{
Tehmina Anjum ${ }^{1,2}$, Wajiha Iram ${ }^{2}\left(\mathbb{D}\right.$, Mazhar Iqbal $^{3}$, Mateen Abbas ${ }^{4}\left(\mathbb{D}\right.$, Waheed Akram ${ }^{1,2,5}$ (D) and Guihua Li ${ }^{1, *}$ \\ 1 Guangdong Key Laboratory for New Technology Research of Vegetables, Vegetable Research Institute, \\ Guangdong Academy of Agricultural Sciences, Guangzhou 510640, China; tehminaanjum@yahoo.com (T.A.); \\ meher_waheed@yahoo.com (W.A.) \\ 2 Department of Plant Pathology, Faculty of Agricultural Sciences, University of the Punjab, \\ Lahore 54000, Pakistan; wajiha_iram@yahoo.com \\ 3 Health Biotechnology Division, National Institute for Biotechnology and Genetic Engineering, \\ Faisalabad 38000, Pakistan; hamzamgondal@yahoo.com \\ 4 Quality Operations Laboratory, Department of Toxicology, University of Veterinary and Animal Sciences, \\ Lahore 54000, Pakistan; mateen.abbas@uvas.edu.pk \\ 5 BECS Analytics and Innovation Research Boulevard, Lahore 54000, Pakistan \\ * Correspondence: liguihua@gdaas.cn
}

Citation: Anjum, T.; Iram, W.; Iqbal, M.; Abbas, M.; Akram, W.; Li, G. Structure Elucidation and Toxicity Analysis of the Byproducts Formed after Biodegradation of Aflatoxins B1 and B2 Using Extracts of Mentha arvensis. Toxins 2022, 14, 24 https://doi.org/10.3390/ toxins 14010024

Received: 5 November 2021 Accepted: 24 December 2021 Published: 1 January 2022

Publisher's Note: MDPI stays neutral with regard to jurisdictional claims in published maps and institutional affiliations.

Copyright: (C) 2022 by the authors. Licensee MDPI, Basel, Switzerland. This article is an open access article distributed under the terms and conditions of the Creative Commons Attribution (CC BY) license (https:// creativecommons.org/licenses/by/ $4.0 /)$.

\begin{abstract}
The aqueous extracts of leaves and shoots of Mentha arvensis were checked for their potential to biodegrade aflatoxin B1 and B2 (AFB1; $100 \mu \mathrm{g} / \mathrm{L}$ and AFB2; $50 \mu \mathrm{g} / \mathrm{L}$ ) through in vitro assays. Overall, the results showed that leaf extract degrades aflatoxins more efficiently than the shoot extract. First, the $\mathrm{pH}$, temperature and incubation time were optimized for maximum degradation by observing this activity at different temperatures between 25 and $60{ }^{\circ} \mathrm{C}$, pH between 2 and 10 and incubation time from 3 to $72 \mathrm{~h}$. In general, an increase in all these parameters significantly increased the percentage of biodegradation. In vitro trials on mature maize stock were performed under optimized conditions, i.e., $\mathrm{pH} 8$, temperature $30{ }^{\circ} \mathrm{C}$ and an incubation period of $72 \mathrm{~h}$. The leaf extract resulted in $75 \%$ and $80 \%$ biodegradation of AFB1 and AFB2, respectively. Whereas the shoot extract degraded both toxins up to $40-48 \%$. The structural elucidation of degraded toxin products by LCMS/MS analysis showed seven degraded products of AFB1 and three of AFB2. MS/MS spectra showed that most of the products were formed by the loss of the methoxy group from the side chain of the benzene ring, the removal of the double bond in the terminal furan ring and the modification of the lactone group, indicating less toxicity compared to the parent compounds. The degraded products showed low toxicity against brine shrimps, confirming that $M$. arvensis leaf extract has significant potential to biodegrade aflatoxins.
\end{abstract}

Keywords: mycotoxins; maize; mass spectrometry; HPLC; Aspergillus flavus

Key Contribution: Bio-degradation of aflatoxins B1 and B2 produced by Aspergillus flavus was performed using aqueous extracts of Mentha arvensis under in vitro conditions.

\section{Introduction}

Aflatoxins comprise a family of extremely toxic mycotoxins produced by Aspergillus flavus, A. parasiticus and $A$. nominus. These are hepatotoxic mycotoxins usually produced in various agricultural commodities. Maize, however, has shown a higher contamination rate as it provides an excellent substrate for mold infection. Among the eighteen different types of aflatoxins identified so far, aflatoxins B1, B2, G1 and G2 have been reported in maize. The order of toxicity is AFB1 > AFG1 > AFB2 > AFG2, probably because of the slight difference in structures of these aflatoxins [1]. AFB1 and AFG1 contain a double bond that 
undergoes reduction, forming vinyl ether at the terminal furan ring; this is not the case in AFB2 and AFG2.

The problem of mycotoxin contamination in maize is more common in tropical and subtropical regions of the world because of hot and humid conditions in these areas. Tabuc, et al. [2] found $30 \%$ of the maize samples contaminated with aflatoxins B1 in a survey between 2002 and 2004 in Southeastern Romania. Similarly, Ghiasian, et al. [3], in 2011, analyzed maize samples from the Kermanshah and Mazandaran provinces of Iran and found aflatoxin contamination between $58 \%$ and $80 \%$. Various surveys have been performed in Pakistan covering the provinces of Punjab and Khaybar Pakhtun Khuah. Scientists have reported aflatoxin levels ranging between 30 and $200 \mu \mathrm{g} / \mathrm{kg}$ [4-7]. In our recent survey encompassing maize storehouses of fifteen districts belonging to three agro-ecological zones of Punjab, Pakistan, 78\% of collected samples were found to be contaminated with aflatoxins B1 and B2. However, aflatoxins G1, G2 and ochratoxin A were not found in any sample [8].

Various physical and chemical methods have been investigated for reducing these carcinogenic toxins to safe levels [9]. Physical methods use binding agents, such as clay, zeolites, sepiolite, kaolin, bentonites, monomorillonite and activated charcoal. The process is expensive and laborious and reduces the toxin to only 50-60\%. A higher inclusion of clay can increase the binding of these compounds with minerals and antibiotics. Most of these binders are not biodegradable; thus, they can cause environmental problems. Chemical methods use caustic soda, ammonia, oxidants (such as ozone, hydrogen peroxide and sodium hypochlorite), reducing agents (such as chlorinated agents, bisulphites and formaldehyde). These chemicals are not safe, hence are not accepted by consumers [10]. Many researchers have tested the potential of microorganisms, including bacteria, yeast and fungi, to degrade these mycotoxins [11]. Microbial degradation of AFB1 using Flavobacterium aurantiacum (now called Nocardia corynebacterioides) was first reported in 1966 [12]. Though detoxification products were not identified, the residual toxicity was found to be absent when checked against ducklings [12]. After this first report, several investigations have concentrated on the biodegradation of AFB1. However, very few reports describe details regarding the degraded products and their toxicity. Many of these studies have shown the transformation of aflatoxins B1 to aflatoxicol, which is eighteen times less toxic than AFB1 but is still hazardous for other organisms [13].

Phytochemicals are of interest as a rich source of natural, ecofriendly antimicrobial components. Recently, many plant sources have been exploited for the detoxification and biodegradation of mycotoxins [14-18]. In this study, we investigated the potential of a common herb, i.e., Mentha arvensis (family: Lamiaceae), to degrade AFB1 and AFB2. Mentha arvensis is a well-known appetizer in Ayurveda and is traditionally used to treat digestive problems and cough [19]. A large number of different chemicals identified in this herb, including $\alpha$-menthol, neomenthol, d-menthone, menthofuran, isomnethone, isomenthol, p-cymene, menthylacetate, cineol, limonine, phellandrene, aromadendrene, $\alpha$ pinene, $\alpha$-phellandrene, pinene, piperitone, carvomenthone, carvacrol, thujone, dipentene, cadinene, menthofuran, linalyl acetate, carvone, linalool and piperitenone oxide, are being used in pharmaceuticals, food, flavoring, cosmetics, beverages and allied industries [20-22]. Earlier studies have also confirmed the antifungal potential of $M$. arvensis [23,24]. Keeping this in view, the present study was planned to explore the biodegrading potential of $M$. arvensis against aflatoxins B1 and B2. The study was extended to the identification of the byproducts and evaluating their biological toxicity.

\section{Results}

2.1. Effect of Temperature on In Vitro Biodegradation of Aflatoxins B1 and B2 by Extracts of Mentha Arvensis

Time course investigation on aflatoxin degradation revealed the start of detoxification within three hours of incubation and a significant increase with an increase in incubation 
time. Qualitative analysis of degraded toxins by thin-layer chromatography showed a distinct decline in florescence with an increase in biodegradation percentage (Figure 1).

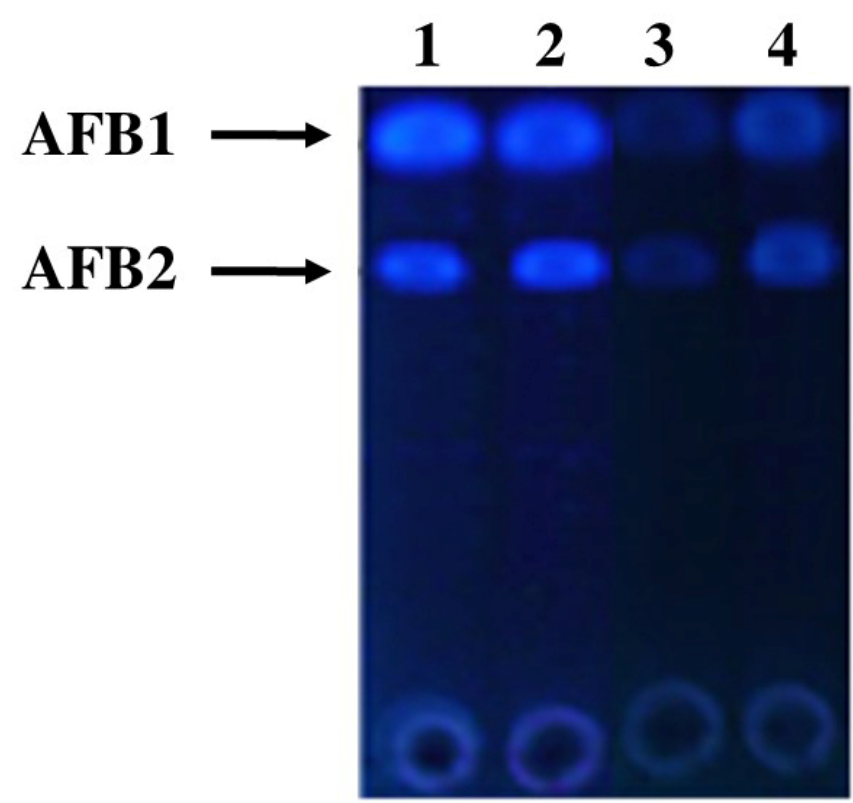

Figure 1. TLC analysis of control and treated toxins at $30^{\circ} \mathrm{C}$ after $72 \mathrm{~h}$ of incubation. Where, $1=$ Control (Toxin); 2 = Control (Toxin + water); 3 = Toxin + Mentha arvensis leaf extract; $4=$ Toxin + Mentha arvensis shoot extract.

At the lowest tested temperature of $25{ }^{\circ} \mathrm{C}$, the leaf extract of $M$. arvensis resulted in $44.31 \%$ and $62.16 \%$ degradation of AFB1 and AFB2, respectively, when checked after $3 \mathrm{~h}$ of incubation. The percentage of degradation increased with an increase in incubation time, as after $72 \mathrm{~h}, 70.9 \%$ of AFB1 and $71.85 \%$ of AFB2 were degraded at $25^{\circ} \mathrm{C}$.

Similarly, an increase in temperature significantly increased the efficacy of $M$. arvensis to biodegrade aflatoxins (Table 1). At $60{ }^{\circ} \mathrm{C}$, the percentage of degradation in both AFB1 and AFB2 was up to $83-88 \%$. Shoot extract was found to be less effective than the leaf extract, as after $72 \mathrm{~h}$ at $60{ }^{\circ} \mathrm{C}$, it degraded AFB1 up to 50.03\% and AFB2 up to 57.46\%. However, further in vitro studies were carried out at $30^{\circ} \mathrm{C}$ to avoid any harmful effect of high temperature on maize. In this trial, the leaf extract of $M$. arvensis at $30^{\circ} \mathrm{C}$ resulted in significant degradation of AFB1 (72.12\%) and AFB2 (74.01\%) after an incubation of $72 \mathrm{~h}$.

\subsection{Effect of $p H$ on In Vitro Biodegradation of Aflatoxins B1 and B2 by Extracts of M. arvensis}

The results present a significant $(p<0.05)$ biodegradation in both aflatoxin B1 and $\mathrm{B} 2$ when incubated at a $\mathrm{pH}$ of 2 . However, an increase in $\mathrm{pH}$ increased this percentage. Similarly, an increase in incubation period also enhanced the biodegradation potential of Mentha arvensis. The leaf extract of $M$. arvensis resulted in more degradation when compared to that of its shoot extract. After three hours of incubation, the leaf extract of M. arvensis caused $43.8 \%$ and $56.9 \%$ degradation of AFB1 and AFB2, respectively, at a $\mathrm{pH}$ of 2. With an increase in $\mathrm{pH}$, this percentage increased, and at the highest tested $\mathrm{pH}$ of 10 a degradation of $69 \%$ was recorded in both AFB1 and AFB2 (Tables 2 and 3). The overall results showed a maximum degradation at $\mathrm{pH} 10$. However, aflatoxins are known to become sensitive and unstable at very high basic $\mathrm{pH}$. Hence to avoid this in further trials, $\mathrm{pH} 8$ was selected, which is much less alkaline than $\mathrm{pH} 10$. The degradation percentages of both AFB1 and AFB2 at pH 8 were also found to be comparable with the results recorded at $\mathrm{pH} 10$. 
Table 1. Effect of Temperature on Biodegradation by Mentha arvensis extracts.

\begin{tabular}{|c|c|c|c|c|c|c|c|c|c|c|c|c|c|}
\hline \multirow{2}{*}{ Treatments } & \multirow{2}{*}{ Temp $\left({ }^{\circ} \mathrm{C}\right)$} & \multicolumn{6}{|c|}{$\%$ Degradation of AFB1 } & \multicolumn{6}{|c|}{$\%$ Degradation of AFB2 } \\
\hline & & $3 \mathrm{~h}$ & $6 \mathrm{~h}$ & $12 \mathrm{~h}$ & $24 \mathrm{~h}$ & $48 \mathrm{~h}$ & $72 \mathrm{~h}$ & $3 \mathrm{~h}$ & $6 \mathrm{~h}$ & $12 \mathrm{~h}$ & $24 \mathrm{~h}$ & $48 \mathrm{~h}$ & $72 \mathrm{~h}$ \\
\hline \multirow{7}{*}{ Toxin } & 25 & $0.3 \pm 0.0^{c}$ & $0.8 \pm 0.0^{c}$ & $1.6 \pm 0.9^{b}$ & $1.9 \pm 0.1^{b}$ & $2.2 \pm 0.1^{\mathrm{a}}$ & $2.9 \pm 0.2^{\mathrm{a}}$ & $0.1 \pm 0.0^{c}$ & $0.5 \pm 0.0^{\mathrm{b}, \mathrm{c}}$ & $0.7 \pm 0.0^{\mathrm{a}-\mathrm{c}}$ & $0.8 \pm 0.0^{\mathrm{a}-\mathrm{c}}$ & $1.0 \pm 0.0^{\mathrm{a}, \mathrm{b}}$ & $1.4 \pm 0.1^{\mathrm{a}}$ \\
\hline & 30 & $0.8 \pm 0.0^{\mathrm{d}}$ & $0.8 \pm 0.0^{\mathrm{d}}$ & $1.8 \pm 0.6^{c}$ & $2.3 \pm 0.1^{b}$ & $3.1 \pm 0.2^{\mathrm{a}}$ & $3.8 \pm 0.1^{\mathrm{a}}$ & $0.1 \pm 0.0^{c}$ & $0.6 \pm 0.0^{b, c}$ & $0.8 \pm 0.0^{b, c}$ & $0.9 \pm 0.1^{\mathrm{a}, \mathrm{b}}$ & $1.1 \pm 0.0^{\mathrm{a}, \mathrm{b}}$ & $1.6 \pm 0.1^{\mathrm{a}}$ \\
\hline & 35 & $1.2 \pm 0.3^{c}$ & $2.5 \pm 0.3^{b, c}$ & $2.5 \pm 0.1^{b, c}$ & $3.0 \pm 0.2^{b}$ & $3.8 \pm 0.2^{\mathrm{a}, \mathrm{b}}$ & $4.5 \pm 0.3^{\mathrm{a}}$ & $0.2 \pm 0.0^{c}$ & $0.8 \pm 0.0^{b, c}$ & $0.8 \pm 0.0^{b, c}$ & $1.1 \pm 0.1^{b}$ & $1.2 \pm 0.1^{\mathrm{a}, \mathrm{b}}$ & $1.8 \pm 0.2^{\mathrm{a}}$ \\
\hline & 40 & $2.2 \pm 0.1^{\mathrm{d}}$ & $3.5 \pm 0.4^{\mathrm{c}}$ & $3.8 \pm 0.2^{c}$ & $4.3 \pm 0.5^{\mathrm{b}}$ & $4.4 \pm 0.3^{b}$ & $5.2 \pm 0.4^{\mathrm{a}}$ & $0.3 \pm 0.0^{c}$ & $0.9 \pm 0.1^{b, c}$ & $0.9 \pm 0.0^{b, c}$ & $1.3 \pm 0.1^{b}$ & $1.4 \pm 0.1^{\mathrm{a}, \mathrm{b}}$ & $2.0 \pm 0.1^{\mathrm{a}}$ \\
\hline & 45 & $3.2 \pm 0.3^{c}$ & $4.5 \pm 0.3^{b}$ & $5.1 \pm 0.3^{a, b}$ & $5.2 \pm 0.4^{\mathrm{a}, \mathrm{b}}$ & $5.6 \pm 0.5^{\mathrm{a}}$ & $5.8 \pm 0.3^{\mathrm{a}}$ & $0.3 \pm 0.0^{\mathrm{c}}$ & $1.0 \pm 0.2^{b, c}$ & $1.1 \pm 0.1^{b, c}$ & $1.4 \pm 0.1^{\mathrm{b}}$ & $1.6 \pm 0.1^{\mathrm{b}}$ & $2.3 \pm 0.2^{\mathrm{a}}$ \\
\hline & 50 & $4.2 \pm 0.5^{c}$ & $5.5 \pm 0.2^{b}$ & $5.8 \pm 0.2^{b}$ & $6.5 \pm 0.3^{a, b}$ & $6.5 \pm 0.4^{\mathrm{a}, \mathrm{b}}$ & $6.9 \pm 0.4^{\mathrm{a}}$ & $0.3 \pm 0.0^{c}$ & $1.1 \pm 0.1^{b, c}$ & $1.2 \pm 0.1^{b}$ & $1.6 \pm 0.1^{b}$ & $1.7 \pm 0.1^{b}$ & $2.5 \pm 0.2^{a}$ \\
\hline & 55 & $5.2 \pm 0.3^{c}$ & $6.4 \pm 0.7^{\mathrm{b}}$ & $6.5 \pm 0.4^{b}$ & $7.1 \pm 0.5^{\mathrm{a}, \mathrm{b}}$ & $7.8 \pm 0.6^{\mathrm{a}}$ & $7.9 \pm 0.6^{\mathrm{a}}$ & $0.4 \pm 0.0^{\mathrm{d}}$ & $1.1 \pm 0.1^{\mathrm{c}}$ & $1.4 \pm 0.1^{b, c}$ & $1.7 \pm 0.1^{\mathrm{b}}$ & $1.9 \pm 0.1^{b}$ & $2.7 \pm 0.1^{a}$ \\
\hline \multirow{8}{*}{$\begin{array}{c}\text { Toxin }+ \\
\mathrm{H}_{2} \mathrm{O}\end{array}$} & 60 & $6.2 \pm 0.4^{b}$ & $7.1 \pm 0.5^{\mathrm{a}, \mathrm{b}}$ & $7.5 \pm 0.8^{a, b}$ & $7.7 \pm 0.8^{\mathrm{a}}$ & $7.8 \pm 0.4^{\mathrm{a}}$ & $7.9 \pm 0.5^{\mathrm{a}}$ & $0.5 \pm 0.0^{\mathrm{d}}$ & $1.2 \pm 0.2^{\mathrm{c}, \mathrm{d}}$ & $1.5 \pm 0.1^{\mathrm{c}}$ & $1.8 \pm 0.2^{b, c}$ & $2.0 \pm 0.1^{b}$ & $3.0 \pm 0.2^{a}$ \\
\hline & 30 & $1.1 \pm 0.1^{\mathrm{d}}$ & $2.5 \pm 0.1^{c}$ & $3.2 \pm 0.3^{b}$ & $3.5 \pm 0.3^{b}$ & $3.8 \pm 0.2^{\mathrm{a}, \mathrm{b}}$ & $4.2 \pm 0.3^{\mathrm{a}}$ & $0.3 \pm 0.0^{\mathrm{d}}$ & $1.1 \pm 0.0^{c}$ & $1.5 \pm 0.0^{c}$ & $2.0 \pm 0.1^{b, c}$ & $2.3 \pm 0.2^{b}$ & $3.4 \pm 0.2^{\mathrm{a}}$ \\
\hline & 35 & $2.4 \pm 0.2^{\mathrm{d}}$ & $3.4 \pm 0.2^{c}$ & $4.7 \pm 0.2^{b}$ & $5.1 \pm 0.3^{a, b}$ & $5.5 \pm 0.3^{\mathrm{a}}$ & $5.7 \pm 0.3^{\mathrm{a}}$ & $1.2 \pm 0.1^{c}$ & $1.2 \pm 0.1^{c}$ & $2.2 \pm 0.1^{b, c}$ & $2.7 \pm 0.2^{\mathrm{a}, \mathrm{b}}$ & $2.8 \pm 0.1^{\mathrm{a}, \mathrm{b}}$ & $3.3 \pm 0.2^{a}$ \\
\hline & 40 & $3.7 \pm 0.2^{\mathrm{d}}$ & $4.7 \pm 0.5^{c}$ & $6.1 \pm 0.5^{b}$ & $6.4 \pm 0.4^{\mathrm{a}, \mathrm{b}}$ & $6.8 \pm 0.7^{\mathrm{a}}$ & $6.8 \pm 0.5^{\mathrm{a}}$ & $2.1 \pm 0.3^{c}$ & $2.7 \pm 0.1^{b}$ & $3.3 \pm 0.2^{a, b}$ & $3.4 \pm 0.2^{a, b}$ & $3.7 \pm 0.2^{a}$ & $3.8 \pm 0.3^{a}$ \\
\hline & 45 & $5.1 \pm 0.4^{\mathrm{d}}$ & $5.9 \pm 0.3^{c}$ & $7.2 \pm 0.6^{b, c}$ & $7.4 \pm 0.5^{b, c}$ & $7.8 \pm 0.5^{b}$ & $8.0 \pm 0.4^{\mathrm{a}}$ & $2.7 \pm 0.2^{\mathrm{d}}$ & $3.9 \pm 0.2^{c}$ & $4.1 \pm 0.3^{b, c}$ & $4.1 \pm 0.3^{b, c}$ & $4.5 \pm 0.1^{\mathrm{a}, \mathrm{b}}$ & $4.9 \pm 0.2^{a}$ \\
\hline & 50 & $6.4 \pm 0.3^{\mathrm{d}}$ & $7.3 \pm 0.6^{c}$ & $8.3 \pm 0.6^{b}$ & $8.9 \pm 0.6^{\mathrm{b}}$ & $9.1 \pm 0.7^{\mathrm{a}, \mathrm{b}}$ & $9.5 \pm 0.8^{a}$ & $3.4 \pm 0.5^{c}$ & $4.4 \pm 0.3^{c}$ & $4.4 \pm 0.1^{\mathrm{c}}$ & $5.6 \pm 0.2^{b}$ & $5.6 \pm 0.4^{b}$ & $6.0 \pm 0.4^{a}$ \\
\hline & 55 & $7.7 \pm 0.6^{\mathrm{d}}$ & $8.7 \pm 0.5^{c}$ & $9.3 \pm 0.8^{b, c}$ & $9.9 \pm 0.7^{b}$ & $10 \pm 1.3^{\mathrm{a}, \mathrm{b}}$ & $10 \pm 0.6^{\mathrm{a}}$ & $4.2 \pm 0.3^{c}$ & $4.8 \pm 0.2^{c}$ & $4.9 \pm 0.3^{c}$ & $6.7 \pm 0.4^{b}$ & $7.1 \pm 0.6^{\mathrm{a}}$ & $7.1 \pm 0.3^{a}$ \\
\hline & 60 & $9.1 \pm 0.7^{\mathrm{d}}$ & $10 \pm 1.1^{\mathrm{c}, \mathrm{d}}$ & $10 \pm 0.9^{c, d}$ & $10 \pm 0.8^{c}$ & $11 \pm 1.1^{\mathrm{b}}$ & $12 \pm 1.1^{\mathrm{a}}$ & $4.9 \pm 0.6^{\mathrm{d}}$ & $5.2 \pm 0.4^{\mathrm{c}, \mathrm{d}}$ & $5.4 \pm 0.4^{c}$ & $7.8 \pm 0.7^{b}$ & $8.3 \pm 0.5^{\mathrm{a}, \mathrm{b}}$ & $8.6 \pm 0.5^{a}$ \\
\hline \multirow{7}{*}{$\begin{array}{c}\text { Toxin + leaf } \\
\text { extract }\end{array}$} & 25 & $44 \pm 2.3^{\mathrm{d}}$ & $46 \pm 2.3^{\mathrm{d}}$ & $52 \pm 3.6^{\mathrm{c}, \mathrm{d}}$ & $60 \pm 4.3^{b, c}$ & $66 \pm 4.3^{\mathrm{a}, \mathrm{b}}$ & $70 \pm 6.3^{\mathrm{a}}$ & $62 \pm 5.6^{c}$ & $65 \pm 4.3^{b, c}$ & $66 \pm 4.6^{\mathrm{b}}$ & $69 \pm 5.7^{a, b}$ & $70 \pm 5.0^{\mathrm{a}}$ & $71 \pm 4.3^{\mathrm{a}}$ \\
\hline & 30 & $49 \pm 4.1^{\mathrm{d}}$ & $52 \pm 4.1^{\mathrm{d}}$ & $58 \pm 2.3^{c, d}$ & $61 \pm 5.1^{b, c}$ & $67 \pm 5.0^{a, b}$ & $72 \pm 5.9^{\mathrm{a}}$ & $63 \pm 7.2^{\mathrm{d}}$ & $65 \pm 5.2^{c, d}$ & $66 \pm 5.2^{c}$ & $70 \pm 6.1^{b}$ & $72 \pm 6.2^{a, b}$ & $74 \pm 6.3^{a}$ \\
\hline & 35 & $53 \pm 3.2^{e}$ & $57 \pm 3.2^{\mathrm{d}, \mathrm{e}}$ & $60 \pm 4.1^{\mathrm{c}, \mathrm{d}}$ & $64 \pm 3.6^{b, c}$ & $68 \pm 3.3^{a, b}$ & $74 \pm 5.0^{\mathrm{a}}$ & $65 \pm 4.2^{\mathrm{d}}$ & $66 \pm 5.3^{c, d}$ & $67 \pm 4.9^{c}$ & $72 \pm 4.8^{b}$ & $74 \pm 5.8^{\mathrm{a}, \mathrm{b}}$ & $75 \pm 5.2^{\mathrm{a}}$ \\
\hline & 45 & $62 \pm 3.7^{\mathrm{e}}$ & $66 \pm 2.9^{\mathrm{d}, \mathrm{e}}$ & $69 \pm 3.7^{c, d}$ & $73 \pm 6.0^{b, c}$ & $79 \pm 4.9^{\mathrm{a}, \mathrm{b}}$ & $85 \pm 6.2^{a}$ & $68 \pm 5.9^{\mathrm{d}}$ & $69 \pm 5.1^{\mathrm{d}}$ & $71 \pm 6.6^{c}$ & $75 \pm 5.3^{b}$ & $77 \pm 4.9^{\mathrm{a}, \mathrm{b}}$ & $78 \pm 5.8^{a}$ \\
\hline & 50 & $63 \pm 4.1^{\mathrm{e}}$ & $66 \pm 4.0^{\mathrm{d}}$ & $70 \pm 6.1^{c}$ & $74 \pm 5.1^{b, c}$ & $80 \pm 5.3^{b}$ & $86 \pm 7.7^{a}$ & $69 \pm 3.7^{d}$ & $72 \pm 4.9^{\mathrm{c}, \mathrm{d}}$ & $73 \pm 5.8^{c}$ & $78 \pm 6.1^{b}$ & $79 \pm 5.7^{a, b}$ & $80 \pm 6.2^{a}$ \\
\hline & 55 & $65 \pm 5.5^{\mathrm{e}}$ & $68 \pm 5.1^{\mathrm{d}}$ & $72 \pm 4.5^{\mathrm{c}, \mathrm{d}}$ & $76 \pm 6.9^{b, c}$ & $82 \pm 4.2^{\mathrm{a}, \mathrm{b}}$ & $87 \pm 4.9^{\mathrm{a}}$ & $71 \pm 4.8^{\mathrm{d}}$ & $72 \pm 2.6^{c, d}$ & $74 \pm 4.8^{b}$ & $79 \pm 5.6^{\mathrm{a}, \mathrm{b}}$ & $79 \pm 5.8^{a, b}$ & $81 \pm 5.4^{\mathrm{a}}$ \\
\hline & 60 & $67 \pm 4.2^{\mathrm{d}}$ & $68 \pm 7.1^{\mathrm{d}}$ & $73 \pm 5.1^{c, d}$ & $78 \pm 5.1^{b, c}$ & $84 \pm 6.7^{a, b}$ & $88 \pm 6.2^{\mathrm{a}}$ & $72 \pm 5.9^{d}$ & $73 \pm 5.9 \mathrm{c}, \mathrm{d}$ & $74 \pm 5.7^{c}$ & $80 \pm 6.2^{b}$ & $82 \pm 6.3^{\mathrm{a}, \mathrm{b}}$ & $83 \pm 7.7^{\mathrm{a}}$ \\
\hline \multirow{8}{*}{$\begin{array}{l}\text { Toxin }+ \\
\text { shoot } \\
\text { extract }\end{array}$} & 25 & $19 \pm 1.3^{\mathrm{d}}$ & $23 \pm 1.9^{c}$ & $25 \pm 2.1^{b, c}$ & $28 \pm 1.3^{b}$ & $30 \pm 2.2^{a, b}$ & $32 \pm 2.6^{\mathrm{a}}$ & $25 \pm 1.9^{f}$ & $29 \pm 1.6^{\mathrm{e}}$ & $32 \pm 1.9^{\mathrm{d}}$ & $38 \pm 2.7^{c}$ & $43 \pm 3.3^{b}$ & $46 \pm 3.6^{a}$ \\
\hline & 30 & $25 \pm 2.2^{d, c}$ & $23 \pm 1.6^{d}$ & $26 \pm 1.8^{c}$ & $29 \pm 1.1^{b, c}$ & $31 \pm 1.9^{b}$ & $34 \pm 2.7^{\mathrm{a}}$ & $26 \pm 2.3^{f}$ & $30 \pm 2.3^{e}$ & $33 \pm 2.0^{\mathrm{d}}$ & $39 \pm 2.8^{c}$ & $45 \pm 2.8^{b}$ & $48 \pm 2.7^{a}$ \\
\hline & 35 & $29 \pm 1.7^{c}$ & $32 \pm 2.5^{b, c}$ & $33 \pm 2.3^{a, b}$ & $35 \pm 2.4^{\mathrm{a}}$ & $36 \pm 3.4^{\mathrm{a}}$ & $36 \pm 3.2^{\mathrm{a}}$ & $28 \pm 1.7^{f}$ & $32 \pm 1.9 \mathrm{~d}, \mathrm{e}$ & $35 \pm 2.2^{\mathrm{d}}$ & $45 \pm 3.3^{c}$ & $48 \pm 3.1^{b}$ & $50 \pm 4.1^{\mathrm{a}}$ \\
\hline & 40 & $36 \pm 1.6^{c}$ & $39 \pm 2.3^{b}$ & $41 \pm 2.9^{\mathrm{a}, \mathrm{b}}$ & $42 \pm 3.2^{a, b}$ & $42 \pm 2.8^{\mathrm{a}}$ & $43 \pm 3.3^{a}$ & $29 \pm 2.2^{\mathrm{e}}$ & $32 \pm 2.6^{\mathrm{d}, \mathrm{e}}$ & $34 \pm 1.7^{\mathrm{d}}$ & $45 \pm 2.1^{c}$ & $49 \pm 3.6^{b}$ & $51 \pm 3.8^{a}$ \\
\hline & 45 & $37 \pm 2.8^{c}$ & $41 \pm 3.7^{b, c}$ & $44 \pm 3.6^{\mathrm{b}}$ & $46 \pm 3.1^{a, b}$ & $46 \pm 3.1^{\mathrm{a}, \mathrm{b}}$ & $47 \pm 2.0^{\mathrm{a}}$ & $31 \pm 1.9^{\mathrm{e}}$ & $33 \pm 1.1^{\mathrm{d}, \mathrm{e}}$ & $35 \pm 1.8^{\mathrm{d}}$ & $42 \pm 1.9^{c}$ & $48 \pm 2.9^{b}$ & $53 \pm 2.7^{a}$ \\
\hline & 50 & $37 \pm 2.7^{c}$ & $41 \pm 2.9^{b, c}$ & $44 \pm 2.8^{b}$ & $46 \pm 2.7^{a, b}$ & $47 \pm 2.9^{a, b}$ & $48 \pm 3.9^{a}$ & $32 \pm 2.8^{\mathrm{e}}$ & $33 \pm 2.7 \mathrm{~d}, \mathrm{e}$ & $35 \pm 2.6^{d}$ & $43 \pm 2.6^{c}$ & $49 \pm 2.7^{b}$ & $54 \pm 3.9^{a}$ \\
\hline & 55 & $40 \pm 3.1^{c}$ & $44 \pm 3.1^{b, c}$ & $45 \pm 3.7^{b}$ & $47 \pm 2.9^{a, b}$ & $48 \pm 3.4^{\mathrm{a}, \mathrm{b}}$ & $49 \pm 2.8^{a}$ & $34 \pm 3.3^{\mathrm{e}}$ & $35 \pm 1.9^{\mathrm{d}, \mathrm{e}}$ & $37 \pm 2.2^{\mathrm{d}}$ & $44 \pm 3.1^{\mathrm{c}}$ & $51 \pm 4.1^{b}$ & $56 \pm 2.5^{a}$ \\
\hline & 60 & $42 \pm 2.8^{c}$ & $45 \pm 2.3^{b, c}$ & $46 \pm 2.6^{\mathrm{b}}$ & $49 \pm 3.2^{a, b}$ & $50 \pm 3.2^{a}$ & $50 \pm 4.0^{\mathrm{a}}$ & $35 \pm 1.2^{\mathrm{e}}$ & $35 \pm 2.3^{\mathrm{d}, \mathrm{e}}$ & $37 \pm 2.9^{\mathrm{d}}$ & $45 \pm 2.3^{c}$ & $52 \pm 4.4^{b}$ & $57 \pm 3.7^{a}$ \\
\hline
\end{tabular}

Data were analyzed by analysis of variance (ANOVA). Letters in upper case indicate significant differences $(p<0.05)$ among tested plant extracts as calculated by Tukey's Multiple

Range test. 
Table 2. Effect of $\mathrm{pH}$ on biodegradation of AFB1 by leaf and shoot extracts of Mentha arvensis.

\begin{tabular}{|c|c|c|c|c|c|c|c|c|c|c|c|c|c|}
\hline \multirow[b]{2}{*}{ Treatments } & \multirow[b]{2}{*}{$\mathrm{pH}$} & \multicolumn{2}{|c|}{$3 \mathrm{~h}$} & \multicolumn{2}{|c|}{$6 \mathrm{~h}$} & \multicolumn{2}{|c|}{$12 \mathrm{~h}$} & \multicolumn{2}{|c|}{$24 \mathrm{~h}$} & \multicolumn{2}{|c|}{$48 \mathrm{~h}$} & \multicolumn{2}{|c|}{$72 \mathrm{~h}$} \\
\hline & & $\begin{array}{c}\text { Toxin } \\
\text { Recovery }\end{array}$ & $\mathrm{D} \%$ & $\begin{array}{c}\text { Toxin } \\
\text { Recovery }\end{array}$ & $\mathrm{D} \%$ & $\begin{array}{c}\text { Toxin } \\
\text { Recovery }\end{array}$ & $\mathrm{D} \%$ & $\begin{array}{c}\text { Toxin } \\
\text { Recovery }\end{array}$ & $\mathrm{D} \%$ & $\begin{array}{c}\text { Toxin } \\
\text { Recovery }\end{array}$ & $\mathrm{D} \%$ & $\begin{array}{c}\text { Toxin } \\
\text { Recovery }\end{array}$ & $\mathrm{D} \%$ \\
\hline Toxin AFB1 & & $99 \pm 7.8^{\mathrm{r}}$ & 0.81 & $99 \pm 6.7^{\mathrm{r}}$ & 0.88 & $98 \pm 7.7^{\mathrm{r}}$ & 1.80 & $97 \pm 5.4^{\mathrm{r}}$ & 2.3 & $96 \pm 6.4^{\mathrm{q}, \mathrm{r}}$ & 3.07 & $96 \pm 7.5^{\mathrm{o}-\mathrm{r}}$ & 3.81 \\
\hline Toxin $+\mathrm{H}_{2} \mathrm{O}$ & $\mathrm{pH} 2$ & $96 \pm 9.5^{p, q, r}$ & 3.2 & $95 \pm 7.3^{\mathrm{n}-\mathrm{r}}$ & 4.6 & $92 \pm 6.9^{\mathrm{k}-\mathrm{p}}$ & 7.8 & $90 \pm 6.9^{g-1}$ & 9.6 & $89 \pm 6.5^{\mathrm{e}-1}$ & 10.2 & $87 \pm 5.9^{\mathrm{d}-\mathrm{j}}$ & 12.7 \\
\hline Toxin $+\mathrm{H}_{2} \mathrm{O}$ & $\mathrm{pH} 4$ & $96 \pm 7.6^{\mathrm{o}-\mathrm{r}}$ & 3.8 & $95 \pm 7.4^{\mathrm{n}-\mathrm{r}}$ & 4.7 & $90 \pm 8.8^{\mathrm{h}-\mathrm{m}}$ & 9.2 & $88 \pm 5.9^{\mathrm{d}-1}$ & 11.6 & $87 \pm 8.9^{\mathrm{d}-\mathrm{i}}$ & 13.0 & $86 \pm 7.9^{\mathrm{c}-\mathrm{h}}$ & 13.9 \\
\hline Toxin $+\mathrm{H}_{2} \mathrm{O}$ & $\mathrm{pH} 6$ & $94 \pm 5.2^{\mathrm{m}-\mathrm{r}}$ & 5.1 & $92 \pm 6.8^{1-q}$ & 7.5 & $90 \pm 6.8^{\mathrm{f}-1}$ & 9.8 & $88 \pm 7.4^{\mathrm{c}-1}$ & 12.0 & $86 \pm 6.1^{c-g}$ & 14.0 & $85 \pm 6.0^{\mathrm{b}-\mathrm{f}}$ & 14.9 \\
\hline Toxin $+\mathrm{H}_{2} \mathrm{O}$ & $\mathrm{pH} 8$ & $91 \pm 6.9^{\mathrm{j}-\mathrm{o}}$ & 8.1 & $89 \pm 6.3^{\mathrm{e}-1}$ & 10.1 & $87 \pm 6.1^{\mathrm{d}-\mathrm{k}}$ & 12.3 & $85 \pm 5.9^{b-c}$ & 14.7 & $84 \pm 5.9^{a-d}$ & 15.9 & $83 \pm 5.7^{\mathrm{a}-\mathrm{d}}$ & 16.5 \\
\hline Toxin $+\mathrm{H}_{2} \mathrm{O}$ & pH10 & $90 \pm 6.8^{\mathrm{i}-\mathrm{n}}$ & 8.6 & $87 \pm 6.1^{\mathrm{d}-\mathrm{k}}$ & 12.2 & $85 \pm 5.0^{b-c}$ & 14.7 & $82 \pm 5.0^{a, b, c}$ & 17.7 & $81 \pm 4.5^{\mathrm{a}, \mathrm{b}}$ & 18.8 & $80 \pm 5.4^{\mathrm{a}}$ & 19.9 \\
\hline Toxin $+\mathrm{H}_{2} \mathrm{O}$ & $\mathrm{WpH}$ & $98 \pm 5.7^{\mathrm{r}}$ & 1.09 & $97 \pm 5.6^{\mathrm{r}}$ & 2.6 & $96 \pm 5.9^{p, q, r}$ & 3.23 & $96 \pm 5.8^{o-r}$ & 3.56 & $96 \pm 5.9^{\mathrm{o}-\mathrm{r}}$ & 3.97 & $95 \pm 7.4^{\mathrm{n}-\mathrm{r}}$ & 4.19 \\
\hline \multirow{6}{*}{ Leaf extract + AFB1 } & $\mathrm{pH} 2$ & $56 \pm 3.3^{\mathrm{h}-\mathrm{u}}$ & 43.8 & $55 \pm 4.4^{\mathrm{c}-\mathrm{u}}$ & 44.4 & $52 \pm 2.2^{\mathrm{a}-\mathrm{t}}$ & 47.7 & $48 \pm 3.0^{\mathrm{a}-\mathrm{o}}$ & 51.7 & $42 \pm 1.8^{a-1}$ & 57.5 & $37 \pm 1.6^{a-j}$ & 62.2 \\
\hline & $\mathrm{pH} 4$ & $54 \pm 2.5^{\mathrm{b}-\mathrm{u}}$ & 45.7 & $55 \pm 3.5^{\mathrm{a}-\mathrm{u}}$ & 44.4 & $52 \pm 3.2^{\mathrm{a}-\mathrm{t}}$ & 47.7 & $48 \pm 1.9^{\mathrm{a}-\mathrm{o}}$ & 51.7 & $42 \pm 2.9^{a-j}$ & 57.5 & $37 \pm 2.5^{a-j}$ & 62.2 \\
\hline & $\mathrm{pH} 6$ & $36 \pm 1.6^{a-q}$ & 64.0 & $34 \pm 2.4^{\mathrm{a}-\mathrm{n}}$ & 65.2 & $30 \pm 1.6^{\mathrm{a}-1}$ & 70.0 & $27 \pm 1.9^{\mathrm{a}-1}$ & 72.6 & $24 \pm 0.9^{\mathrm{a}-\mathrm{i}}$ & 75.9 & $20 \pm 0.6^{\mathrm{a}-\mathrm{e}}$ & 79.6 \\
\hline & $\mathrm{pH} 8$ & $38 \pm 3.7^{\mathrm{a}-\mathrm{n}}$ & 61.6 & $34 \pm 1.3^{a-1}$ & 65.7 & $30 \pm 1.5^{\mathrm{a}-\mathrm{k}}$ & 69.4 & $28 \pm 1.1^{a-j}$ & 71.2 & $23 \pm 0.7^{\mathrm{a}-\mathrm{h}}$ & 76.8 & $18 \pm 0.4^{\mathrm{a}, \mathrm{b}, \mathrm{c}}$ & 81.8 \\
\hline & pH10 & $30 \pm 1.5^{a-j}$ & 69.1 & $28 \pm 1.2^{a-j}$ & 72.0 & $26 \pm 1.9^{a-h}$ & 73.9 & $23 \pm 0.8^{\mathrm{a}-\mathrm{g}}$ & 76.4 & $17 \pm 1.5^{\mathrm{a}, \mathrm{b}}$ & 82.4 & $12 \pm 0.3^{\mathrm{a}}$ & 87.6 \\
\hline & $\mathrm{WpH}$ & $57 \pm 3.4^{\mathrm{i}-\mathrm{u}}$ & 42.5 & $56 \pm 3.3^{\mathrm{d}-\mathrm{u}}$ & 43.6 & $53 \pm 3.4^{\mathrm{b}-\mathrm{t}}$ & 46.3 & $50 \pm 4.2^{\mathrm{a}-\mathrm{q}}$ & 49.8 & $45 \pm 3.1^{\mathrm{a}-\mathrm{m}}$ & 54.4 & $40 \pm 1.9^{\mathrm{a}-1}$ & 59.3 \\
\hline \multirow{5}{*}{ Shoot extract + AFB1 } & $\mathrm{pH} 2$ & $75 \pm 4.7^{\mathrm{q}-\mathrm{u}}$ & 24.7 & $76 \pm 4.6^{\mathrm{r}-\mathrm{u}}$ & 23.7 & $75 \pm 6.5^{q-u}$ & 24.2 & $72 \pm 5.5^{\mathrm{k}-\mathrm{u}}$ & 27.2 & $66 \pm 4.3^{\mathrm{e}-\mathrm{u}}$ & 34.0 & $61 \pm 3.6^{\mathrm{d}-\mathrm{u}}$ & 38.1 \\
\hline & $\mathrm{pH} 4$ & $77 \pm 5.8^{\mathrm{p}-\mathrm{u}}$ & 22.6 & $76 \pm 4.7^{\mathrm{p}-\mathrm{u}}$ & 23.4 & $76 \pm 4.7^{\mathrm{o}-\mathrm{u}}$ & 24.0 & $69 \pm 4.2^{\mathrm{f}-\mathrm{u}}$ & 30.8 & $68 \pm 3.5^{\mathrm{e}-\mathrm{u}}$ & 31.2 & $63 \pm 5.5^{\mathrm{d}-\mathrm{u}}$ & 36.3 \\
\hline & $\mathrm{pH} 6$ & $74 \pm 4.3^{\mathrm{k}-\mathrm{u}}$ & 25.9 & $73 \pm 6.2^{\mathrm{j}-\mathrm{u}}$ & 26.7 & $71 \pm 4.3^{\mathrm{g}-\mathrm{u}}$ & 28.5 & $69 \pm 3.2^{\mathrm{f}-\mathrm{u}}$ & 30.3 & $67 \pm 3.8^{\mathrm{e}-\mathrm{u}}$ & 32.4 & $62 \pm 2.5^{\mathrm{d}-\mathrm{u}}$ & 37.1 \\
\hline & pH8 & $67 \pm 3.8^{\mathrm{e}-\mathrm{u}}$ & 32.1 & $66 \pm 3.9^{\mathrm{e}-\mathrm{u}}$ & 33.6 & $65 \pm 2.6^{\mathrm{e}-\mathrm{u}}$ & 34.6 & $64 \pm 4.1^{\mathrm{d}-\mathrm{u}}$ & 35.8 & $60 \pm 3.5^{b-u}$ & 39.3 & $57 \pm 3.2^{\mathrm{a}-\mathrm{u}}$ & 42.8 \\
\hline & $\mathrm{WpH}$ & $73 \pm 4.4^{\mathrm{j}-\mathrm{u}}$ & 26.2 & $71 \pm 4.5^{\mathrm{g}-\mathrm{u}}$ & 28.8 & $68 \pm 3.8^{\mathrm{f}-\mathrm{u}}$ & 31.4 & $65 \pm 5.1^{\mathrm{e}-\mathrm{u}}$ & 34.6 & $62 \pm 3.7^{\mathrm{f}-\mathrm{u}}$ & 37.3 & $59 \pm 4.3^{\mathrm{j}-\mathrm{u}}$ & 40.2 \\
\hline
\end{tabular}

Data were analyzed by analysis of variance (ANOVA). Letters in uppercase indicate significant differences $(p<0.05)$ as calculated by Tukey's Multiple Range test. D\%: Degradation percentage. $\mathrm{WpH}$ : without $\mathrm{pH}$ adjustment. 
Table 3. Effect of $\mathrm{pH}$ on biodegradation of AFB2 by leaf and shoot extracts of Mentha arvensis.

\begin{tabular}{|c|c|c|c|c|c|c|c|c|c|c|c|c|c|}
\hline \multirow[b]{2}{*}{ Treatments } & \multirow[b]{2}{*}{$\mathrm{pH}$} & \multicolumn{2}{|c|}{$3 \mathrm{~h}$} & \multicolumn{2}{|c|}{$6 \mathrm{~h}$} & \multicolumn{2}{|c|}{$12 \mathrm{~h}$} & \multicolumn{2}{|c|}{$24 \mathrm{~h}$} & \multicolumn{2}{|c|}{$48 \mathrm{~h}$} & \multicolumn{2}{|c|}{$72 \mathrm{~h}$} \\
\hline & & $\begin{array}{c}\text { Toxin } \\
\text { Recovery }\end{array}$ & $\mathrm{D} \%$ & $\begin{array}{c}\text { Toxin } \\
\text { Recovery }\end{array}$ & $\mathrm{D} \%$ & $\begin{array}{c}\text { Toxin } \\
\text { Recovery }\end{array}$ & $\mathrm{D} \%$ & $\begin{array}{c}\text { Toxin } \\
\text { Recovery }\end{array}$ & $\mathrm{D} \%$ & $\begin{array}{c}\text { Toxin } \\
\text { Recovery }\end{array}$ & $\mathrm{D} \%$ & $\begin{array}{c}\text { Toxin } \\
\text { Recovery }\end{array}$ & $\mathrm{D} \%$ \\
\hline Toxin AFB1 & & $49 \pm 2^{n}$ & 0.17 & $49 \pm 2^{1-n}$ & 0.68 & $49 \pm 2^{k-n}$ & 0.79 & $49 \pm 3^{h-n}$ & 0.99 & $49 \pm 1^{g-n}$ & 1.16 & $49 \pm 2^{d-1}$ & 1.67 \\
\hline Toxin $+\mathrm{H}_{2} \mathrm{O}$ & $\mathrm{pH} 2$ & $49 \pm 1^{\mathrm{n}}$ & 1.7 & $49 \pm 2^{\mathrm{m}, \mathrm{n}}$ & 1.3 & $49 \pm 7^{\mathrm{k}-\mathrm{n}}$ & 4.0 & $49 \pm 3^{j-n}$ & 4.2 & $49 \pm 3^{i-n}$ & 5.8 & $49 \pm 3^{g-n}$ & 5.4 \\
\hline Toxin $+\mathrm{H}_{2} \mathrm{O}$ & $\mathrm{pH} 4$ & $49 \pm 3^{m, n}$ & 2.8 & $49 \pm 3^{1-n}$ & 3.3 & $49 \pm 3^{e-n}$ & 6.8 & $49 \pm 4^{\mathrm{d}-\mathrm{n}}$ & 7.1 & $49 \pm 2^{\mathrm{d}-\mathrm{m}}$ & 7.6 & $49 \pm 2^{d-1}$ & 9.7 \\
\hline Toxin $+\mathrm{H}_{2} \mathrm{O}$ & $\mathrm{pH} 6$ & $49 \pm 3^{f-n}$ & 6.5 & $49 \pm 2^{\mathrm{f}-\mathrm{n}}$ & 6.4 & $48 \pm 3^{c-k}$ & 10.9 & $48 \pm 2^{c-i}$ & 10.9 & $49 \pm 3^{c-h}$ & 10.3 & $48 \pm 3^{c-h}$ & 11.0 \\
\hline Toxin $+\mathrm{H}_{2} \mathrm{O}$ & $\mathrm{pH} 8$ & $49 \pm 2^{d-m}$ & 7.8 & $48 \pm 3^{b-g}$ & 11.4 & $48 \pm 2^{a-g}$ & 13.0 & $48 \pm 3^{a-e}$ & 12.8 & $48 \pm 1^{\mathrm{a}-\mathrm{e}}$ & 11.7 & $48 \pm 3^{\mathrm{a}-\mathrm{d}}$ & 12.9 \\
\hline Toxin $+\mathrm{H}_{2} \mathrm{O}$ & $\mathrm{pH} 10$ & $48 \pm 4^{\mathrm{a}-\mathrm{f}}$ & 12.3 & $48 \pm 3^{a-c}$ & 15.4 & $48 \pm 4^{\mathrm{a}-\mathrm{c}}$ & 15.5 & $48 \pm 3^{a-c}$ & 15.6 & $48 \pm 2^{a, b}$ & 17.5 & $48 \pm 3^{a}$ & 17.2 \\
\hline Toxin $+\mathrm{H}_{2} \mathrm{O}$ & $\mathrm{WpH}$ & $49 \pm 2^{m, n}$ & 0.36 & $49 \pm 3^{g-n}$ & 1.14 & $49 \pm 3^{d-m}$ & 1.56 & $48 \pm 3^{c-j}$ & 2.04 & $48 \pm 3^{\mathrm{a}-\mathrm{f}}$ & 2.41 & $48 \pm 2^{\mathrm{a}, \mathrm{b}}$ & 3.41 \\
\hline \multirow{6}{*}{ Leaf extract + AFB1 } & $\mathrm{pH} 2$ & $21 \pm 1^{\mathrm{e}-\mathrm{s}}$ & 56.9 & $20 \pm 1^{d-p}$ & 59.9 & $18 \pm 1^{c-p}$ & 62.9 & $17 \pm 1^{\mathrm{b}-\mathrm{o}}$ & 65.8 & $15 \pm 1^{b-p}$ & 68.8 & $16 \pm 1^{b-n}$ & 67.7 \\
\hline & $\mathrm{pH} 4$ & $21 \pm 1^{\mathrm{e}-\mathrm{q}}$ & 58.0 & $18 \pm 1^{c-p}$ & 62.6 & $17 \pm 1^{\mathrm{b}-\mathrm{o}}$ & 64.7 & $16 \pm 1^{b-n}$ & 66.5 & $16 \pm 1^{b-n}$ & 67.3 & $13 \pm 0^{a-f}$ & 72.5 \\
\hline & $\mathrm{pH} 6$ & $19 \pm 2^{d-p}$ & 60.3 & $18 \pm 1^{\mathrm{c}-\mathrm{p}}$ & 62.6 & $16 \pm 1^{\mathrm{b}-\mathrm{n}}$ & 66.7 & $16 \pm 1^{\mathrm{b}-\mathrm{n}}$ & 67.4 & $15 \pm 0^{\mathrm{b}-\mathrm{p}}$ & 69.6 & $13 \pm 1^{\mathrm{a}-\mathrm{e}}$ & 74.1 \\
\hline & $\mathrm{pH} 8$ & $17 \pm 1^{\mathrm{b}-\mathrm{o}}$ & 65.5 & $16 \pm 1^{b-n}$ & 67.8 & $13 \pm 1^{\mathrm{a}-\mathrm{f}}$ & 73.0 & $12 \pm 1^{\mathrm{a}-\mathrm{e}}$ & 75.2 & $12 \pm 1^{\mathrm{a}-\mathrm{e}}$ & 76.0 & $07 \pm 0.6^{\mathrm{a}, \mathrm{b}}$ & 84.1 \\
\hline & $\mathrm{pH} 10$ & $15 \pm 1^{b-1}$ & 69.5 & $13 \pm 1^{\mathrm{a}-\mathrm{f}}$ & 72.5 & $10 \pm 0^{a-d}$ & 79.2 & $9.0 \pm 0^{a-c}$ & 81.9 & $08 \pm 0.3^{\mathrm{a}, \mathrm{b}}$ & 83.6 & $07 \pm 0.4^{\mathrm{a}}$ & 85.2 \\
\hline & $\mathrm{WpH}$ & $18 \pm 0^{\mathrm{c}-\mathrm{p}}$ & 63.2 & $19 \pm 2^{d-p}$ & 61.0 & $17 \pm 1^{\mathrm{b}-\mathrm{o}}$ & 64.8 & $16 \pm 1^{b-n}$ & 66.4 & $15 \pm 1^{\mathrm{b}-\mathrm{p}}$ & 69.2 & $13 \pm 1^{\mathrm{a}-\mathrm{f}}$ & 72.8 \\
\hline \multirow{5}{*}{ Shoot extract + AFB1 } & $\mathrm{pH} 2$ & $39 \pm 2^{y}$ & 21.7 & $37 \pm 2^{x, y}$ & 24.9 & $35 \pm 2^{v-y}$ & 28.4 & $35 \pm 2^{u-y}$ & 29.9 & $34 \pm 2^{s-y}$ & 30.9 & $32 \pm 2^{q-y}$ & 35.1 \\
\hline & $\mathrm{pH} 4$ & $37 \pm 3^{x, y}$ & 24.6 & $35 \pm 1^{\mathrm{u}-\mathrm{y}}$ & 29.3 & $32 \pm 2^{q-y}$ & 35.0 & $30 \pm 3^{p-y}$ & 38.8 & $29 \pm 1^{1-x}$ & 42.0 & $26 \pm 1^{j-x}$ & 46.2 \\
\hline & $\mathrm{pH} 6$ & $36 \pm 2^{v-y}$ & 27.8 & $33 \pm 2^{r-y}$ & 32.5 & $30 \pm 1^{p-y}$ & 38.2 & $29 \pm 1^{1-x}$ & 41.9 & $27 \pm 2^{j-x}$ & 45.1 & $25 \pm 1^{h-x}$ & 49.3 \\
\hline & $\mathrm{pH} 8$ & $35 \pm 2^{u-y}$ & 29.2 & $33 \pm 2^{q-y}$ & 34.0 & $30 \pm 2^{n-y}$ & 39.7 & $28 \pm 2^{k-x}$ & 43.4 & $26 \pm 1^{i-x}$ & 46.6 & $24 \pm 1^{g-x}$ & 50.8 \\
\hline & $\mathrm{WpH}$ & $36 \pm 2^{w-y}$ & 27.6 & $34 \pm 2^{s-y}$ & 31.8 & $33 \pm 2 q-y$ & 33.2 & $32 \pm 2^{q-y}$ & 35.6 & $30 \pm 2^{p-y}$ & 38.8 & $28 \pm 1^{\mathrm{k}-\mathrm{x}}$ & 43.0 \\
\hline
\end{tabular}

Data were analyzed by analysis of variance (ANOVA). Letters in upper case indicate significant differences $(p<0.05)$ among tested plant extracts, as calculated by Tukey's Multiple Range test. $\mathrm{D} \%$ : degradation percentage. $\mathrm{WpH}$ : without $\mathrm{pH}$ 


\subsection{In Vitro Biodegradation of Aflatoxin B1 and B2 in Maize Samples}

A similar trend of degradation was recorded in in vitro trials using mature maize stock. This study was conducted in optimized conditions, i.e., a $\mathrm{pH}$ of 8 , temperature of $30{ }^{\circ} \mathrm{C}$ and an incubation time of $72 \mathrm{~h}$. In the control, maize samples were spiked with $100 \mu \mathrm{g} / \mathrm{L}$ AFB1 and $50 \mu \mathrm{g} / \mathrm{L}$ AFB2; the toxin recovery was 97.3 and $47.6 \mu \mathrm{g} / \mathrm{L}$. When the spiked maize was treated with leaf extract of $M$. arvensis, the toxin recovery reduced to $24.9 \mu \mathrm{g} / \mathrm{L}$ for AFB1 and $9.6 \mu \mathrm{g} / \mathrm{L}$ for AFB2, respectively. The potential of the shoot extract was found to be lower than the leaf extract. Its percentage biodegradation was $40 \%$ in the case of AFB1 and 46.5\% for AFB2 (Table 4).

Table 4. In vitro biodegradation of AFB1 and AFB2 at $\mathrm{pH} 8$ and $30^{\circ} \mathrm{C}$ after $72 \mathrm{~h}$ of incubation.

\begin{tabular}{ccc}
\hline Treatments & \multicolumn{2}{c}{ Toxin Recovery $(\mu \mathrm{g} / \mathrm{L})$} \\
\cline { 2 - 3 } & AFB1 & AFB2 \\
\hline Unspiked maize & $0.5 \pm 0.02^{\mathrm{f}}$ & $00.3 \pm 0.01^{\mathrm{e}}$ \\
Unspiked maize + leaf extract & $00.0 \pm 0.0^{\mathrm{g}}$ & $00.0 \pm 0.0^{\mathrm{f}}$ \\
Unspiked maize + shoot extract & $00.0 \pm 0.0^{\mathrm{g}}$ & $00.0 \pm 0.0^{\mathrm{f}}$ \\
Spiked maize with AFB1 (100 $\mu \mathrm{g} / \mathrm{L})$ and AFB2 $(50 \mu \mathrm{g} / \mathrm{L})$ & $97.3 \pm 6.7^{\mathrm{a}}$ & $47.6 \pm 2.4^{\mathrm{b}}$ \\
Spiked maize with toxin + leaf extract & $24.9 \pm 1.3^{\mathrm{e}}$ & $09.6 \pm 0.8^{\mathrm{d}}$ \\
Degradation \% & $75.1 \pm 4.9^{\mathrm{b}}$ & $80.8 \pm 5.9^{\mathrm{a}}$ \\
Spiked maize with toxin + shoot extract & $60.0 \pm 3.8^{\mathrm{c}}$ & $26.7 \pm 1.3^{\mathrm{c}}$ \\
Degradation \% & $40.0 \pm 2.5^{\mathrm{d}}$ & $46.5 \pm 1.8^{\mathrm{b}}$ \\
\hline
\end{tabular}

Data were analyzed by analysis of variance (ANOVA). Letters in upper case indicate significant differences $(p<0.05)$ among tested plant extracts, as calculated by Tukey's Multiple Range test.

\subsection{Mass Spectral Identification of Degraded Products of AFB1 and AFB2 Treated with M. arvensis Leaf Extracts}

The degradation products of AFB1 and AFB2 in response to the treatment with $M$. arvensis leaf extract showed structural alteration in the parent compound. The structural formulas of the identified degraded products of AFB1 and AFB2 are shown in Figure 2A,B. Leaf extract degraded aflatoxin B1 into seven new compounds and aflatoxin B2 into three products.

\subsection{MS/MS Analysis for Confirmation of AFB1degraded Products}

The degraded products at $m / z 279.17$ and $m / z 283.08$ corresponded to molecular formulas $\mathrm{C}_{16} \mathrm{H}_{6} \mathrm{O}_{5}$ and $\mathrm{C}_{16} \mathrm{H}_{10} \mathrm{O}_{5}$. Both products were obtained by the loss of the methoxy group from the side chain of the benzene ring, with a difference of four hydrogen atoms. The double bond equivalence (DBE) of $\mathrm{C}_{16} \mathrm{H}_{10} \mathrm{O}_{5}$ was the same as that of AFB1, while $\mathrm{C}_{16} \mathrm{H}_{6} \mathrm{O}_{5}$ showed a DBE content greater than AFB1, i.e., 14. The loss of carbon monoxide and oxygen was the main fragmentation pathway of both products. More detail on the fragmentation of precursor ions is shown in Figures 3 and 4.

Similarly, the degraded products obtained at $295.08\left(\mathrm{C}_{16} \mathrm{H}_{22} \mathrm{O}_{5}\right)$ and $293.17 \mathrm{~m} / \mathrm{z}$ $\left(\mathrm{C}_{16} \mathrm{H}_{20} \mathrm{O}_{5}\right)$ were produced by the loss of $\mathrm{CO}$ from the lactone ring of the parent compound. The difference between the two products is of two hydrogen atoms with the same DBE content less than AFB1, i.e., 6. MS/MS analysis of the precursor ion $\mathrm{C}_{16} \mathrm{H}_{22} \mathrm{O}_{5}$ showed product ions represented by 277.17[M- $\left.\mathrm{H}_{2} \mathrm{O}\right]+, 267.08[\mathrm{M}-\mathrm{CO}]+, 253.08\left[\mathrm{M}-\mathrm{CO}_{2}\right]+$, 239.0[M-C $\left.\mathrm{C}_{2} \mathrm{O}_{2}\right]+$ and 225.17 $\left[\mathrm{M}-\mathrm{C}_{2} \mathrm{O}_{3}\right]+(+2 \mathrm{H})$. While precursor ion $\mathrm{C}_{16} \mathrm{H}_{20} \mathrm{O}_{5}$ yielded a series of product ions represented by $275.17\left[\mathrm{M}-\mathrm{H}_{2} \mathrm{O}\right]+, 265.08[\mathrm{M}-\mathrm{CO}]+, 251.08\left[\mathrm{M}-\mathrm{C}_{2} \mathrm{H}_{2 \mathrm{O}}\right]+$ and 231.17[M- $\left.\mathrm{C}_{2} \mathrm{H}_{6} \mathrm{O}_{2}\right]+$ (Figures 5 and 6). 
A

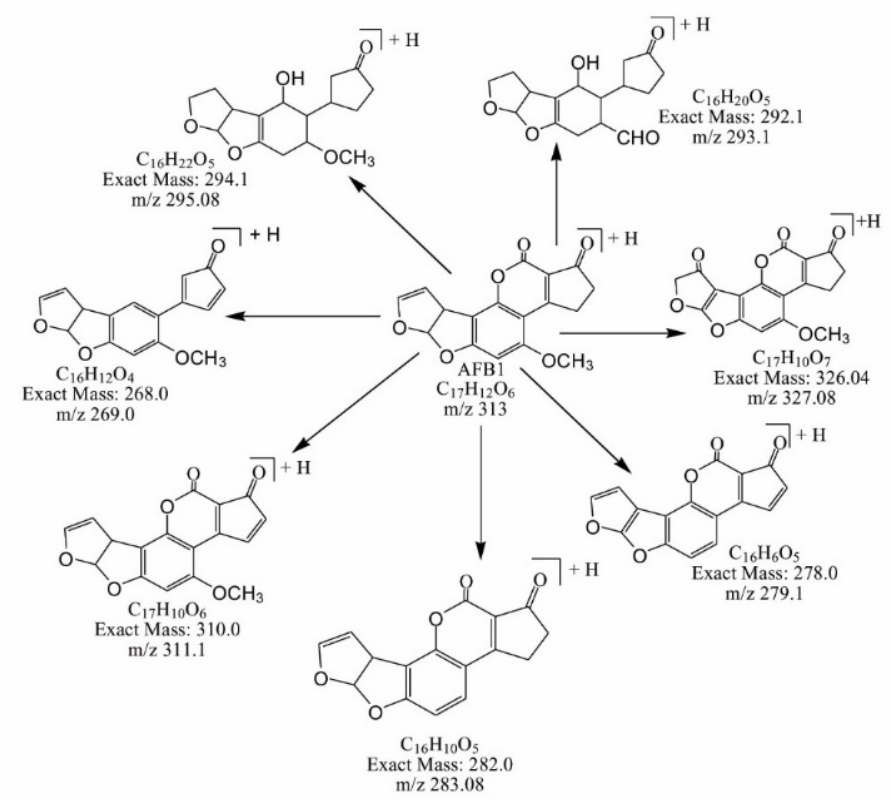

B

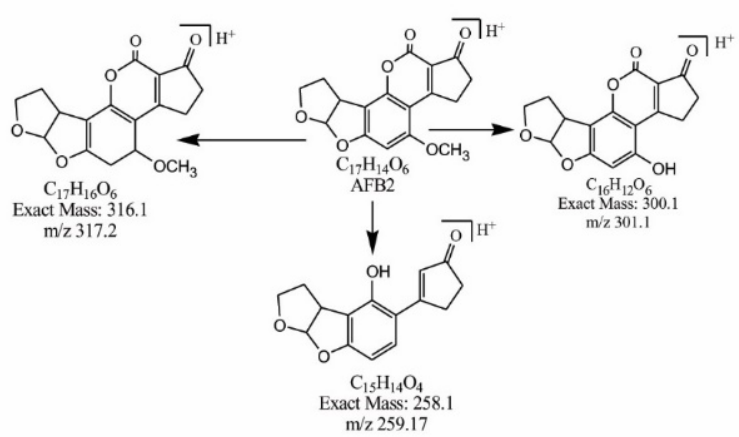

Figure 2. Identified degraded products of (A) AFB1 and (B) AFB2 after incubation with leaf extracts of $M$. arvensis.
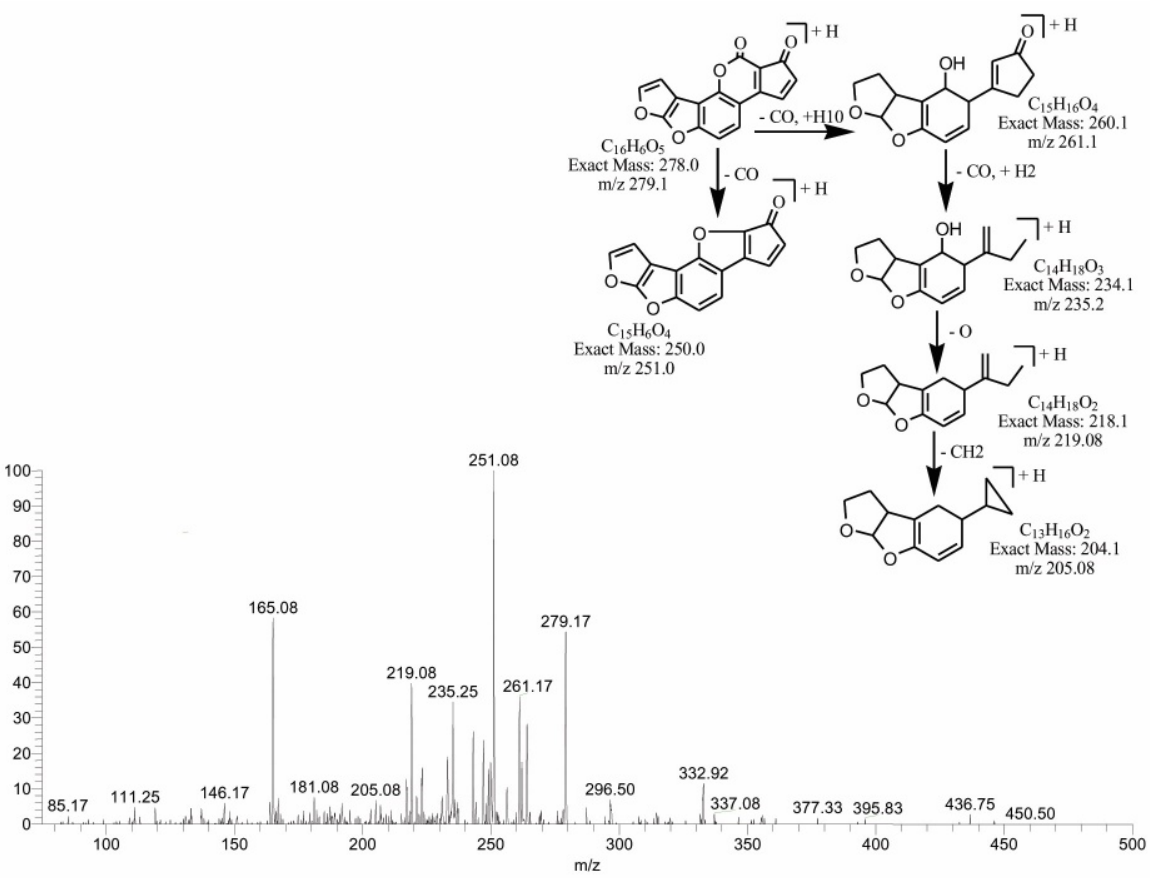

Figure 3. MS/MS spectra and fragmentation pathway of degradation product with $279.17 \mathrm{~m} / \mathrm{z}$. 


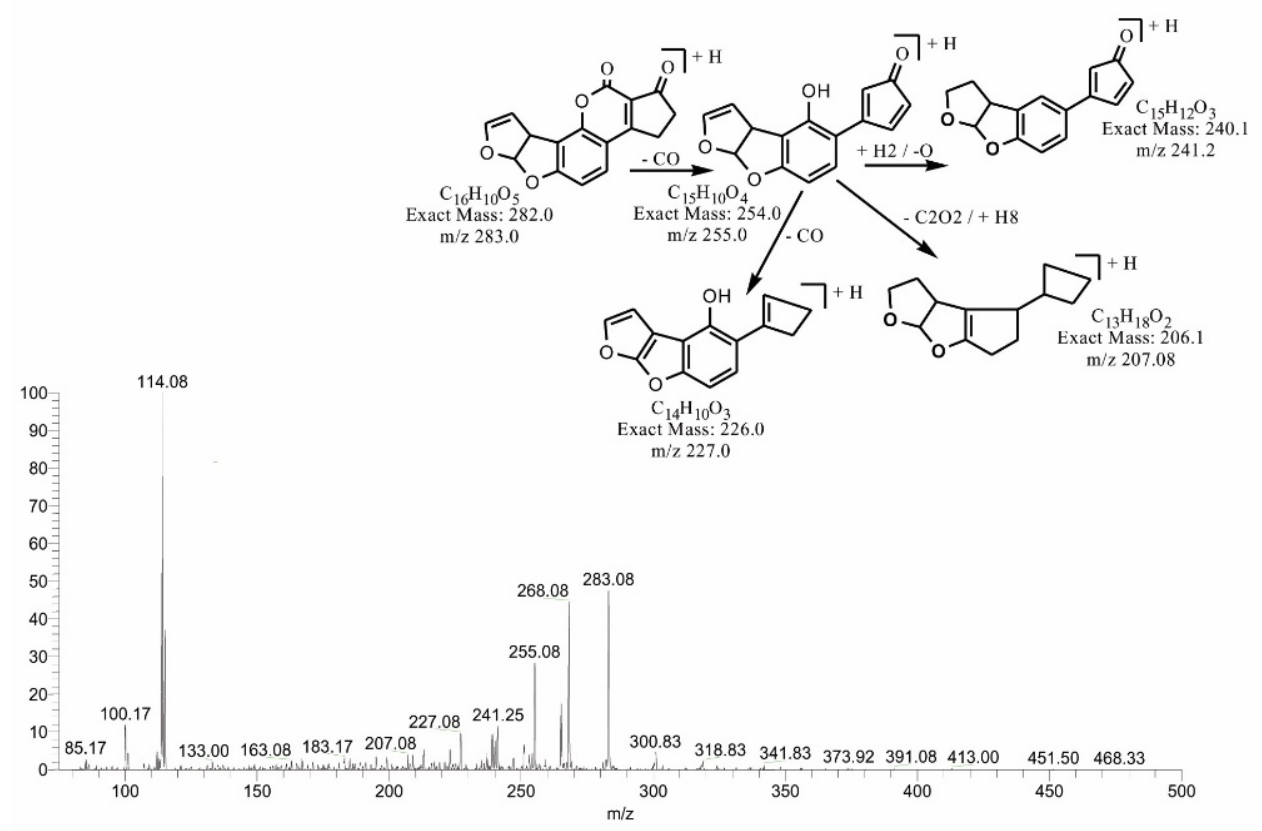

Figure 4. MS/MS spectra and fragmentation pathway of degraded product with $283.08 \mathrm{~m} / \mathrm{z}$.

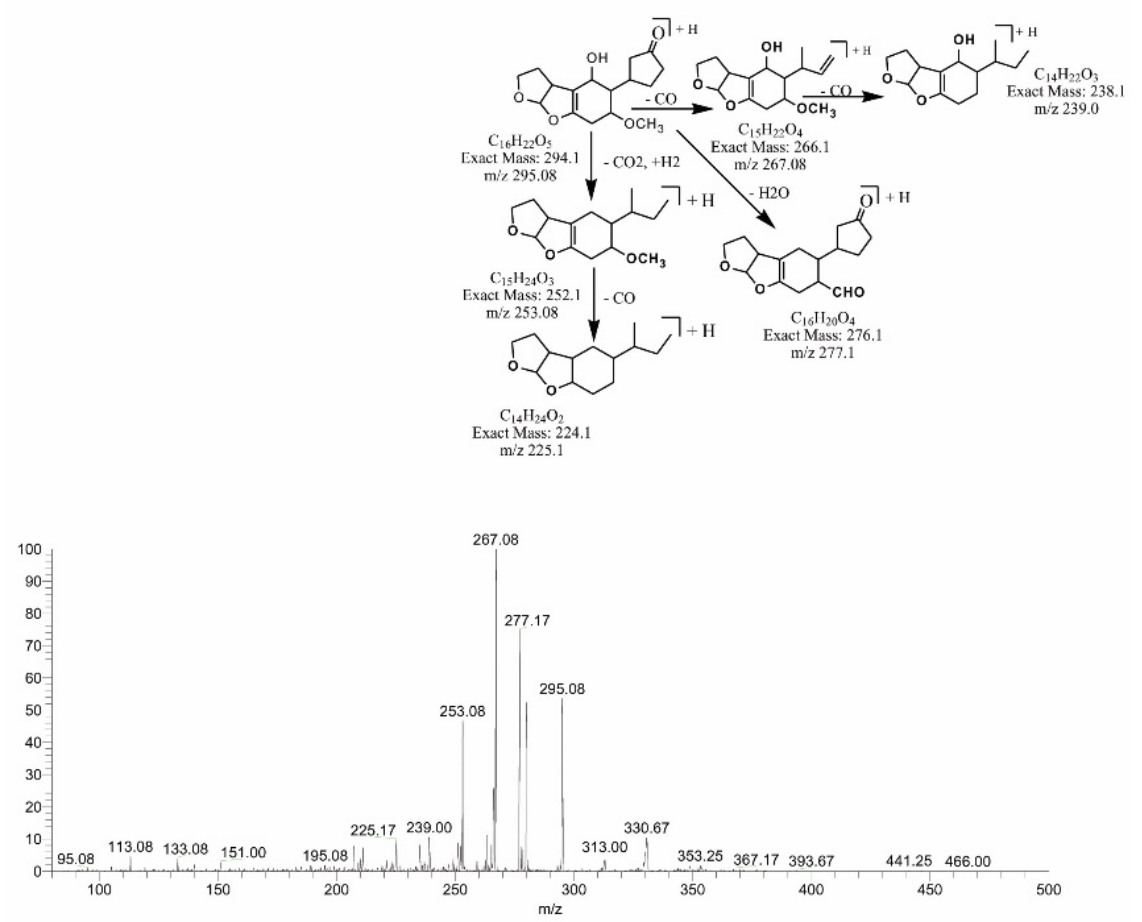

Figure 5. MS/MS spectra and fragmentation pathway of degraded product with $295.08 \mathrm{~m} / \mathrm{z}$.

The degradation product $\mathrm{C}_{16} \mathrm{H}_{12} \mathrm{O}_{4}$ (with $269.00 \mathrm{~m} / \mathrm{z}$ ) was formed by the loss of carbon dioxide from the lactone ring. The $\mathrm{DBE}$ of $\mathrm{C}_{16} \mathrm{H}_{12} \mathrm{O}_{4}$ was one less than AFB1. Loss of $\mathrm{CO}$ was the main fragmentation pathway. More details on the fragmentation pathway are shown in Figure 7. While the product obtained at $311.17 \mathrm{~m} / z\left(\mathrm{C}_{17} \mathrm{H}_{10} \mathrm{O}_{6}\right)$ had two more hydrogen atoms than AFB1, the DBE content of $\mathrm{C}_{17} \mathrm{H}_{10} \mathrm{O}_{6}$ was one more than AFB1, i.e., 13. Fragments of the precursor ion showed losses of $\mathrm{CO}, \mathrm{CH}_{2}$ and $\mathrm{O}$ (Figure 8). The degradation product $\mathrm{C}_{17} \mathrm{H}_{10} \mathrm{O}_{7}$ (with $m / z$ 327.0) was formed by the addition of an oxygen atom onto the double bond of the furan ring; the DBE content was greater than AFB1, i.e., 13. The precursor ion yielded a series of product ions, i.e., $[\mathrm{M}-\mathrm{CO}]+,\left[\mathrm{M}-\mathrm{CO}_{2}\right]+$ and $\left[\mathrm{M}-\mathrm{C}_{2} \mathrm{O}_{2}\right]+$ (Figure 9). 


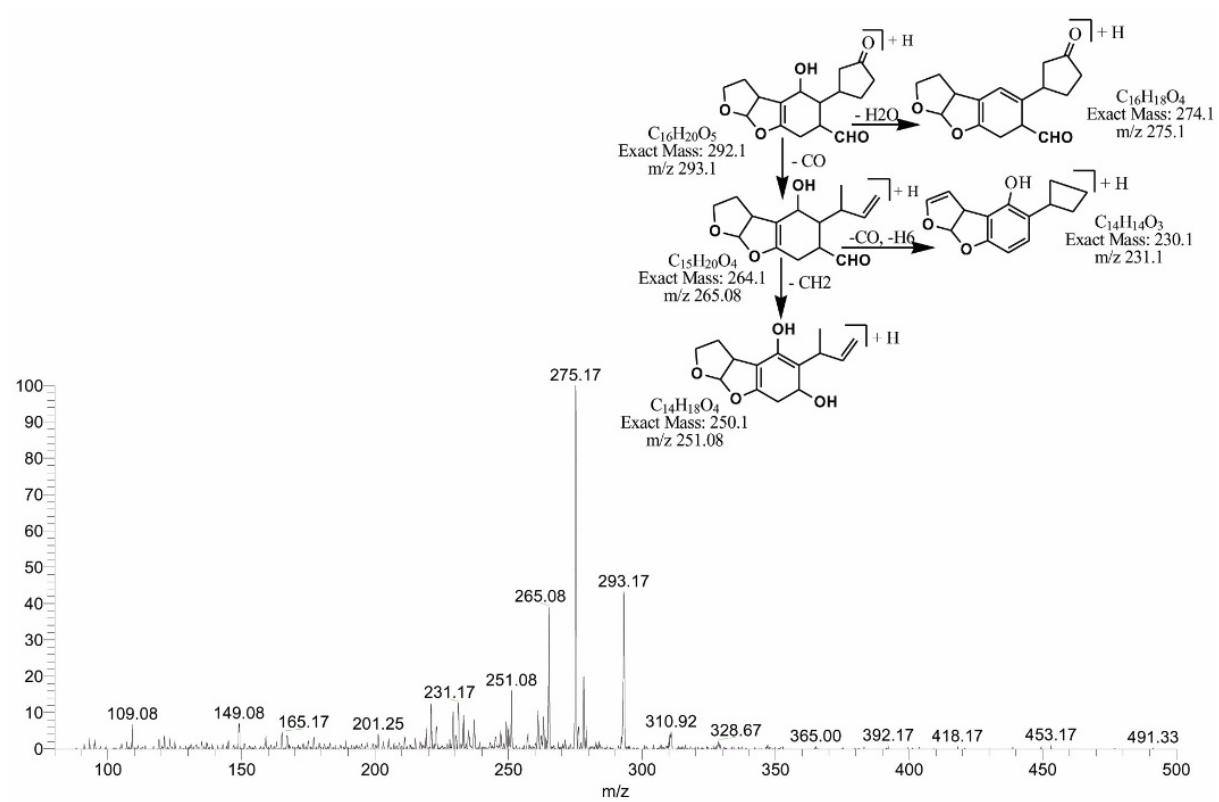

Figure 6. MS/MS spectra and fragmentation pathway of degraded product with $293.17 \mathrm{~m} / \mathrm{z}$.

\subsection{MS/MS Analysis for Confirmation of Degraded Products of AFB2}

The degraded products of AFB2 obtained at 317.25, 301.17 and $259.17 \mathrm{~m} / \mathrm{z}$ corresponded to molecular formulas $\mathrm{C}_{17} \mathrm{H}_{16} \mathrm{O}_{6}, \mathrm{C}_{16} \mathrm{H}_{12} \mathrm{O}_{6}$ and $\mathrm{C}_{15} \mathrm{H}_{14} \mathrm{O}_{4}$, respectively. The product $\mathrm{C}_{17} \mathrm{H}_{16} \mathrm{O}_{6}$ was formed by the addition of two hydrogen atoms to the AFB2 molecule with DBE content less than that of AFB2, i.e., 10. The fragmentation pathway of $\mathrm{C}_{17} \mathrm{H}_{16} \mathrm{O}_{6}$ showed that precursor ions yielded a series of product ions, i.e., 299.25[M-CH 6$]+, 281.25[\mathrm{M}-$ $\left.\mathrm{CH}_{8} \mathrm{O}\right]+, 255.17\left[\mathrm{M}-\mathrm{C}_{2} \mathrm{H}_{6} \mathrm{O}_{2}\right]+$ and 213.08[M- $\left.\mathrm{C}_{3} \mathrm{H}_{4} \mathrm{O}_{4}\right]+$ (Figure 10).

Similarly, by the replacement of the methoxy group with the hydroxyl group on the side chain of the benzene ring, degradation product $\mathrm{C}_{16} \mathrm{H}_{12} \mathrm{O}_{6}$ was formed. The DBE of $\mathrm{C}_{16} \mathrm{H}_{12} \mathrm{O}_{6}$ was the same as that of AFB2. Fragments of $301.17 \mathrm{~m} / \mathrm{z}$ showed losses of $\mathrm{H}_{2} \mathrm{O}, \mathrm{CO}_{2}$ and $\mathrm{CO}$ (Figure 11). The degradation product $\mathrm{C}_{15} \mathrm{H}_{14} \mathrm{O}_{4}$ was originated by the removal of $\mathrm{CO}$ from the lactone ring of $\mathrm{AFB} 2$, with a DBE content less than AFB2, i.e., 9. The loss of $\mathrm{CO}$ and $\mathrm{H}_{2} \mathrm{O}$ was the main fragmentation pathway of $\mathrm{C}_{15} \mathrm{H}_{14} \mathrm{O}_{4}$ (Figure 12).

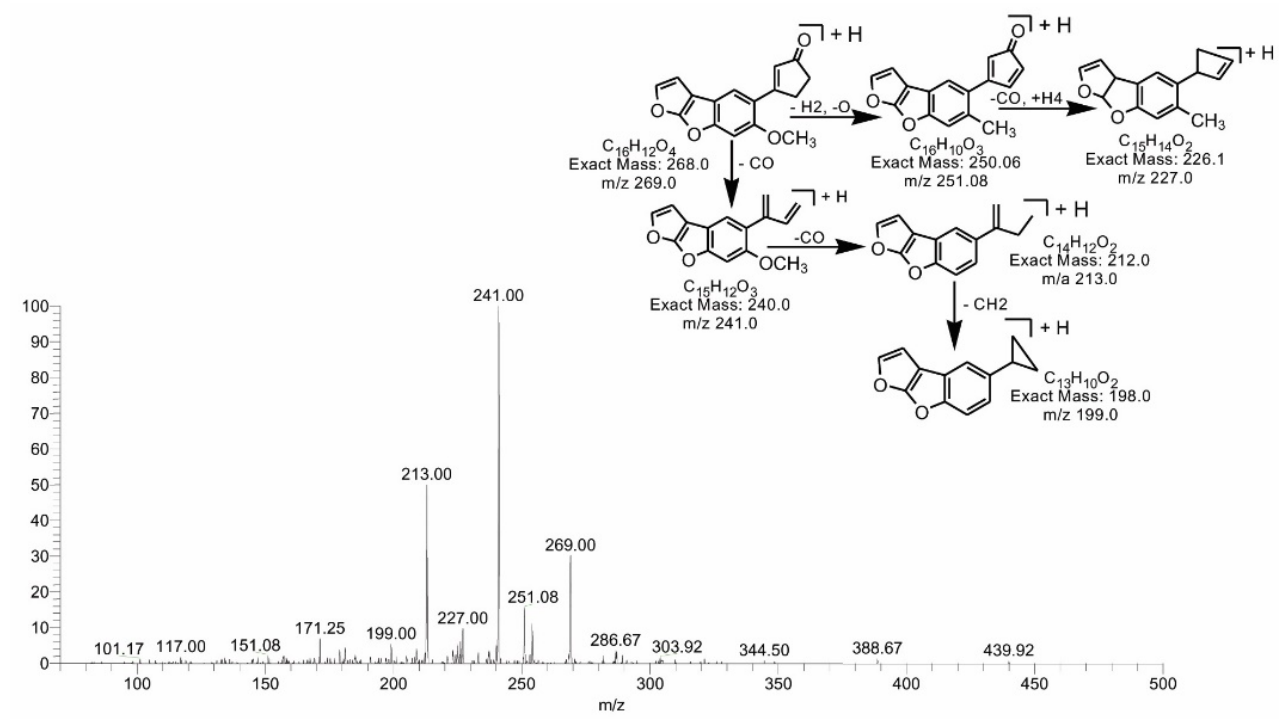

Figure 7. MS/MS spectra and fragmentation pathway of degraded product with $269.00 \mathrm{~m} / \mathrm{z}$. 

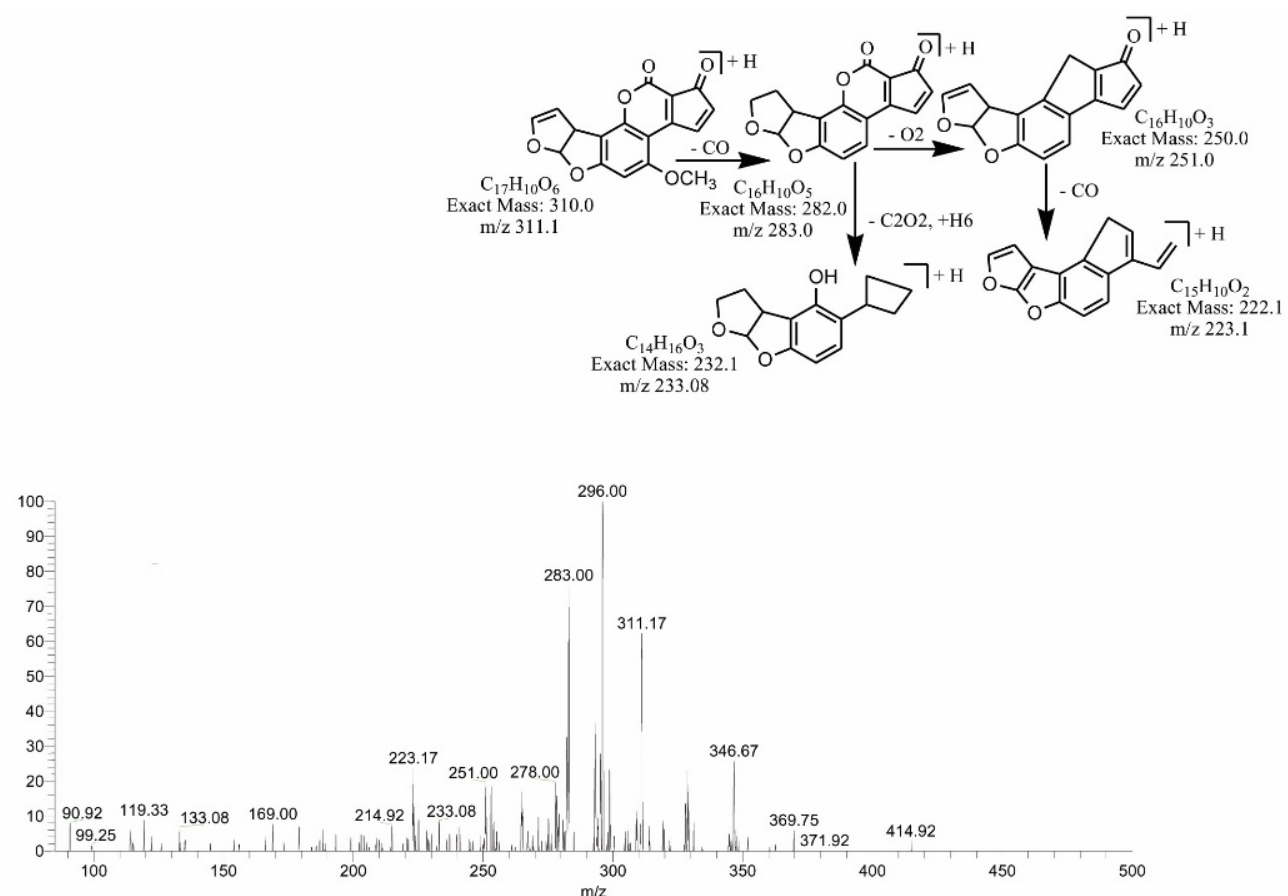

Figure 8. MS/MS spectra and fragmentation pathway of degraded product with $311.17 \mathrm{~m} / \mathrm{z}$.
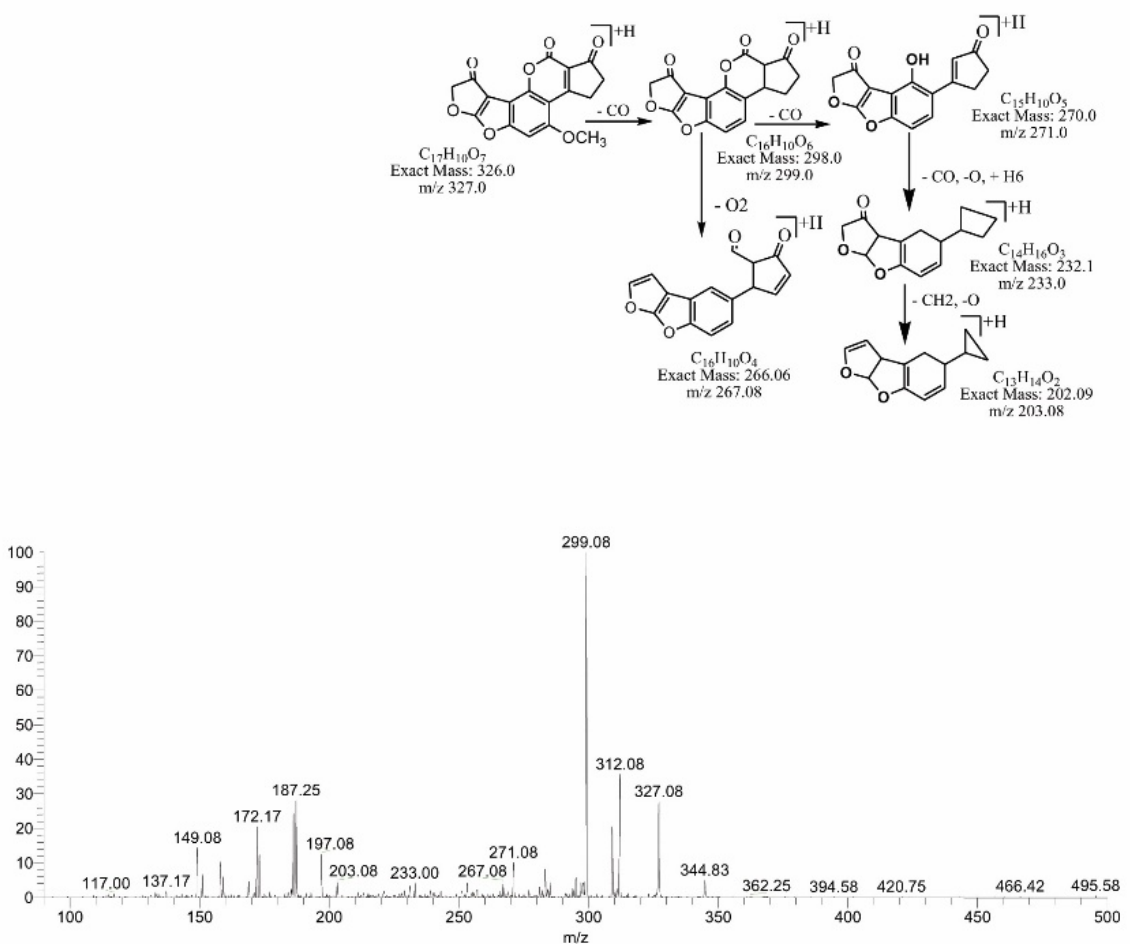

Figure 9. MS/MS spectra and fragmentation pathway of degraded product with $327.08 \mathrm{~m} / \mathrm{z}$. 


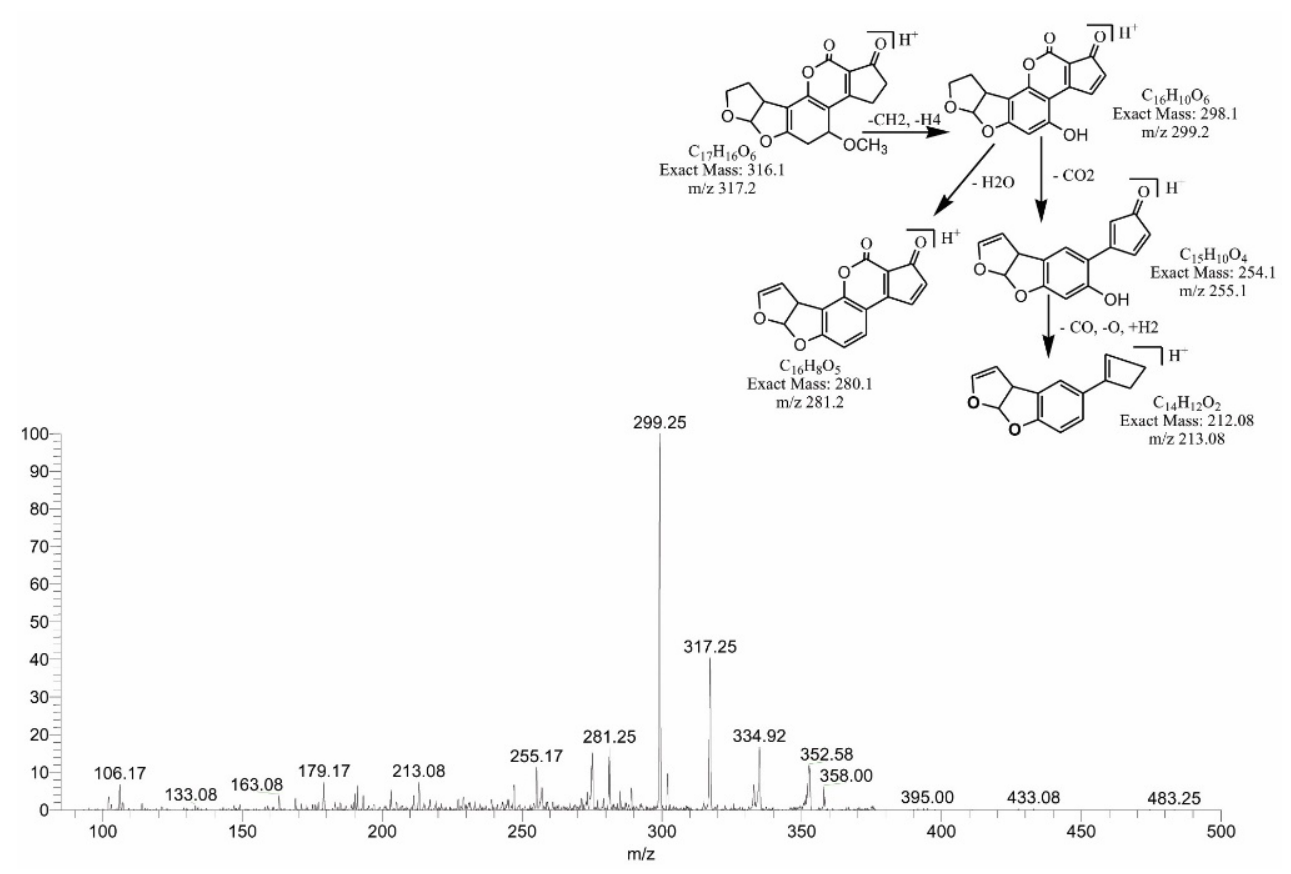

Figure 10. MS/MS spectra and fragmentation pathway of degraded product with $317.25 \mathrm{~m} / z$.

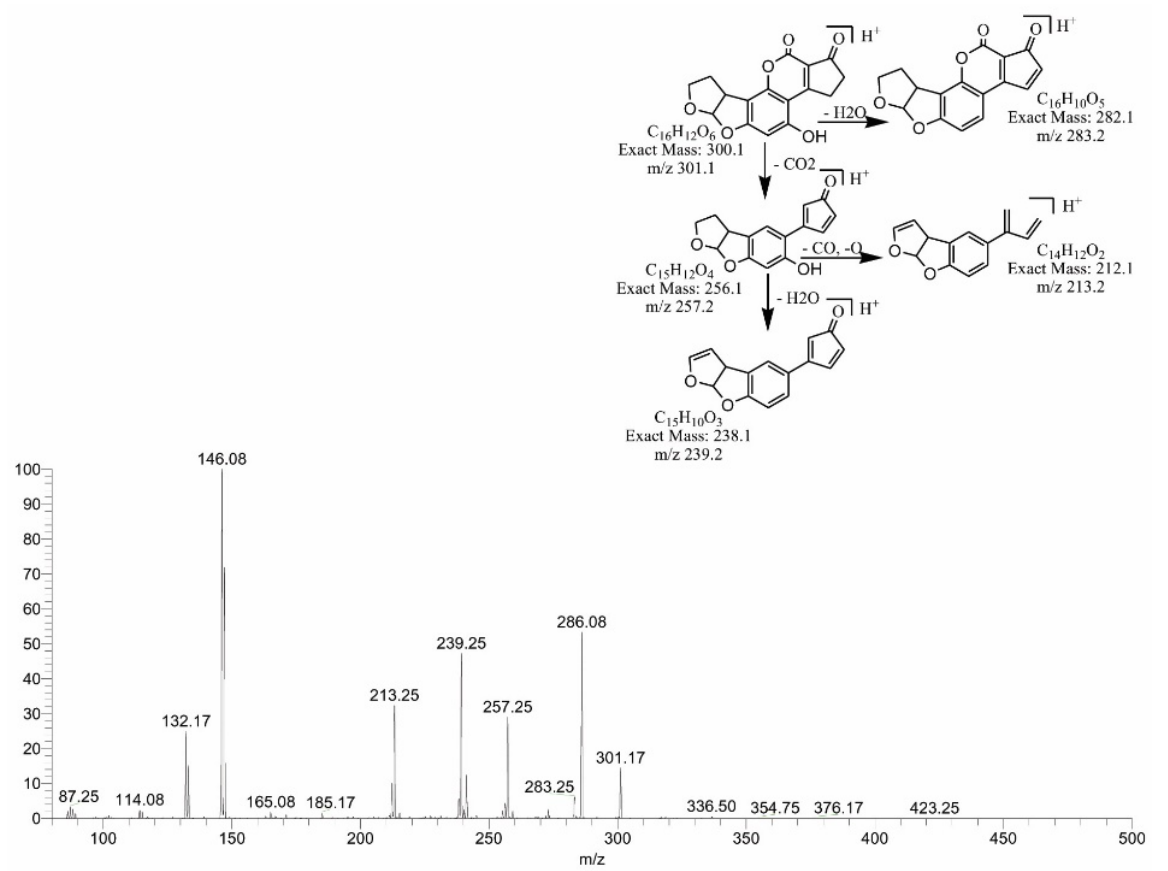

Figure 11. MS/MS spectra and fragmentation pathway of degraded product with $301.17 \mathrm{~m} / \mathrm{z}$. 

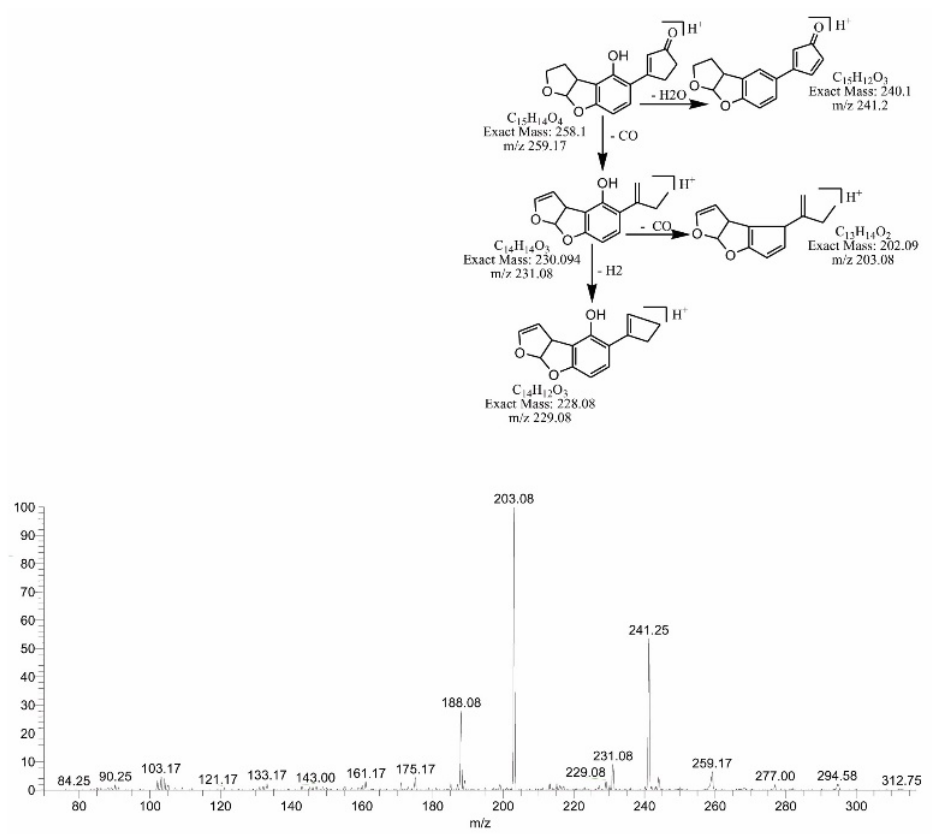

Figure 12. MS/MS spectra and fragmentation pathway of degraded product with $259.17 \mathrm{~m} / \mathrm{z}$.

\subsection{Assessment of Biological Toxicity of Degraded Products}

At the lowest tested concentration of AFB1 (50 $\mu \mathrm{g} / \mathrm{L})$ and AFB2 $(20 \mu \mathrm{g} / \mathrm{L})$, a significant increase in larval mortality was observed compared to the control, i.e., $75 \%$. This percentage of mortality was increased to $87.5 \%$ with an increase in incubation time. The mortality response of larvae varied according to the dosage concentration in an experimented period of 24 to 96 h. Larvae treated with dosage concentrations of 100 (AFB1), 50 (AFB2), 200 (AFB1) and $90 \mu \mathrm{g} / \mathrm{L}$ (AFB2) showed 83-91.7\% and 86.7-96.7\% mortality after 24 to $96 \mathrm{~h}$ of incubation, respectively. Similarly, at the highest tested concentration of $300 \mu \mathrm{g} / \mathrm{L}$ AFB1 and $130 \mu \mathrm{g} / \mathrm{L}$ AFB2, the percentage of larval mortality was found to be $95-100 \%$ after 24 to $96 \mathrm{~h}$, respectively (Table 5).

A significant reduction in larval mortality was recorded after incubation with treated toxins in comparison to the ones incubated with untreated toxins (Figure 13). Larvae incubated with treated $100 \mu \mathrm{g} / \mathrm{L}$ of AFB1 and $50 \mu \mathrm{g} / \mathrm{L}$ of AFB2 showed a percentage mortality of $19.2 \%$ after $24 \mathrm{~h}$ that increased to $28.3 \%$ after $96 \mathrm{~h}$ of incubation. This percentage mortality was $63 \%$ lower than when shrimps were incubated in a similar concentration of untreated toxins. 
Table 5. Percent mortality of brine shrimp larvae at $26^{\circ} \mathrm{C}$ after 24 to $96 \mathrm{~h}$ at various dose levels of treated and untreated aflatoxins.

\begin{tabular}{|c|c|c|c|c|c|c|c|c|c|c|c|c|c|c|}
\hline \multirow{2}{*}{ Treatments } & \multicolumn{2}{|c|}{ Toxin Conc. $\mu \mathrm{g} / \mathrm{L}$} & \multicolumn{4}{|c|}{ No. of Living Cells (hours) } & \multicolumn{4}{|c|}{ No. of Dead Cells (hours) } & \multicolumn{4}{|c|}{$\begin{array}{l}\begin{array}{c}\% \text { Mortality } \\
\text { (hours) }\end{array} \\
\end{array}$} \\
\hline & AFB1 & AFB2 & 24 & 48 & 72 & 96 & 24 & 48 & 72 & 96 & 24 & 48 & 72 & 96 \\
\hline \multirow{2}{*}{$\begin{array}{l}\text { Seawater + s srimps } \\
\text { Methanol + shrimps }\end{array}$} & - & - & $40 \pm 2.1^{\mathrm{a}}$ & $40 \pm 2.5^{\mathrm{a}}$ & $40 \pm 2.8^{\mathrm{a}}$ & $39 \pm 1.3^{\mathrm{a}}$ & $0.1 \pm 0.0^{\mathrm{f}}$ & $0.6 \pm 0.0^{\mathrm{e}}$ & $0.5 \pm 0.0^{\mathrm{e}}$ & $1.1 \pm 0.0^{\mathrm{e}}$ & $0.2 \pm 0.0^{\mathrm{f}}$ & $0.1 \pm 0.0^{\mathrm{e}}$ & $0.2 \pm 0.0^{\mathrm{e}}$ & $2.5 \pm 0.1^{\mathrm{e}, \mathrm{f}}$ \\
\hline & - & - & $38 \pm 2.3^{\mathrm{a}}$ & $38 \pm 1.9^{a}$ & $37 \pm 2.0^{b}$ & $36 \pm 2.8^{a}$ & $1.6 \pm 0.0^{\mathrm{e}}$ & $2.2 \pm 0.1^{\mathrm{d}}$ & $3.1 \pm 0.2^{d}$ & $3.5 \pm 0.1^{\mathrm{d}}$ & $2.5 \pm 0.1^{\mathrm{e}}$ & $5.3 \pm 0.3^{d}$ & $7.5 \pm 0.4^{\mathrm{d}}$ & $7.5 \pm 0.6^{\mathrm{e}}$ \\
\hline \multirow{3}{*}{$\begin{array}{c}\text { Untreated } \\
\text { toxins + shrimps }\end{array}$} & 100 & 50 & $7.3 \pm 0.6^{b, c}$ & $5.4 \pm 0.3^{c}$ & $4.5 \pm 3.4^{c}$ & $3.7 \pm 1.5^{\mathrm{b}, \mathrm{c}}$ & $33 \pm 1.1^{b, c}$ & $35 \pm 3.1 \mathrm{a}, \mathrm{b}$ & $36 \pm 1.8 \mathrm{a}, \mathrm{b}$ & $37 \pm 1.1 \mathrm{a}, \mathrm{b}$ & $83 \pm 3.8^{\mathrm{b}, \mathrm{c}}$ & $86 \pm 7.3^{a, b}$ & $89 \pm 5.0^{a, b}$ & $91 \pm 5.2^{b, c}$ \\
\hline & 200 & 90 & $5.9 \pm 0.2^{c, d}$ & $3.2 \pm 0.1^{c}$ & $2.9 \pm 1.2^{d}$ & $1.9 \pm 1.0^{\mathrm{c}, \mathrm{d}}$ & $35 \pm 2.8 \mathrm{a}, \mathrm{b}$ & $37 \pm 1.6^{a}$ & $38 \pm 1.7^{\mathrm{a}}$ & $39 \pm 2.5^{a}$ & $86 \pm 5.9^{a, b}$ & $91 \pm 5.4^{\mathrm{a}}$ & $94 \pm 6.3^{\mathrm{a}}$ & $96 \pm 5.7^{b}$ \\
\hline & 300 & 130 & $2.3 \pm 0.1^{\mathrm{e}}$ & $2.8 \pm 0.1^{c, d}$ & $1.7 \pm 0.8^{\mathrm{e}}$ & $0.3 \pm 0.0^{\mathrm{e}}$ & $38 \pm 1.7^{\mathrm{a}}$ & $38 \pm 2.5^{\mathrm{a}}$ & $39 \pm 2.9^{\mathrm{a}}$ & $40 \pm 3.1^{\mathrm{a}}$ & $95 \pm 7.1^{\mathrm{a}}$ & $95 \pm 3.6^{\mathrm{a}}$ & $96 \pm 5.8^{a}$ & $100 \pm 7^{a}$ \\
\hline \multirow{3}{*}{$\begin{array}{l}\text { Toxin degraded with } \\
\text { M. arvensis Leaf } \\
\text { extracts + shrimps }\end{array}$} & 50 & 20 & $35 \pm 2.4^{\mathrm{a}}$ & $33 \pm 2.6^{\mathrm{a}}$ & $32 \pm 2.1^{\mathrm{a}}$ & $31 \pm 1.7^{\mathrm{a}}$ & $05 \pm 0.3^{b, c}$ & $07 \pm 0.3^{b, c}$ & $08 \pm 0.6^{b, c}$ & $09 \pm 0.5^{b, c}$ & $13 \pm 1.4^{\mathrm{d}}$ & $17 \pm 1.3^{\mathrm{d}}$ & $19 \pm 1.4^{\mathrm{d}}$ & $22 \pm 1.5^{\mathrm{c}, \mathrm{d}}$ \\
\hline & 100 & 50 & $32 \pm 1.9^{\mathrm{a}}$ & $31 \pm 1.7^{\mathrm{a}}$ & $31 \pm 1.1^{\mathrm{a}}$ & $29 \pm 1.4^{\mathrm{a}}$ & $08 \pm 0.7^{b}$ & $09 \pm 0.5^{b}$ & $09 \pm 0.1^{\mathrm{b}}$ & $11 \pm 0.7^{\mathrm{b}}$ & $19 \pm 1.0^{c}$ & $21 \pm 1.4^{\mathrm{c}}$ & $23 \pm 1.9^{c}$ & $28 \pm 1.4^{c}$ \\
\hline & 300 & 130 & $26 \pm 1.9^{\mathrm{b}, \mathrm{c}}$ & $25 \pm 2.2^{c}$ & $25 \pm 0.9^{b}$ & $24 \pm 1.5^{b, c}$ & $14 \pm 1.1^{\mathrm{a}}$ & $15 \pm 1.1^{\mathrm{a}}$ & $15 \pm 1.1^{\mathrm{a}}$ & $16 \pm 1.3^{a}$ & $34 \pm 2.7^{\mathrm{a}}$ & $36 \pm 2.8^{\mathrm{a}}$ & $38 \pm 2.6^{a}$ & $40 \pm 2.7 \mathrm{a}$ \\
\hline
\end{tabular}

Data were analyzed by analysis of variance (ANOVA). Letters in upper case indicate significant differences $(p<0.05)$ among tested plant extracts, as calculated by Tukey's Multiple Range test. 
$\mathbf{A}$

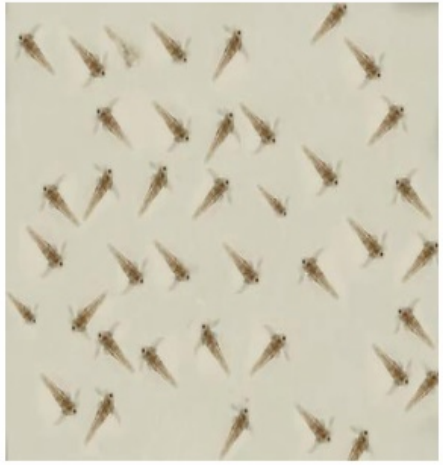

$\mathbf{C}$

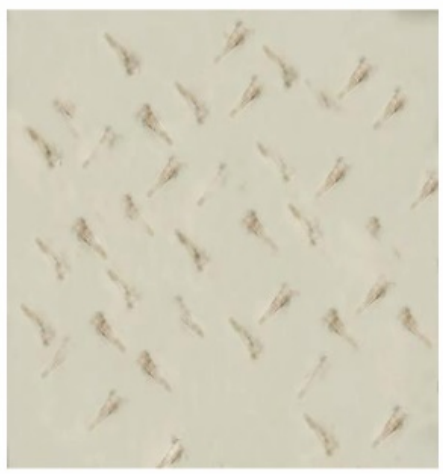

B

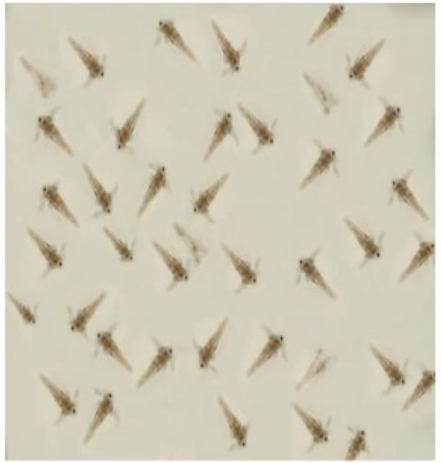

D

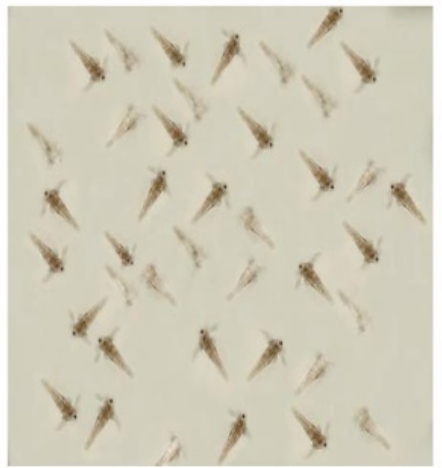

Figure 13. Comparison of toxicity of treated and untreated toxins at concentrations of $300 \mu \mathrm{g} / \mathrm{L}$ (AFB1) and $130 \mu \mathrm{g} / \mathrm{L}$ (AFB2) towards brine shrimp larvae after $96 \mathrm{~h}$ of incubation. The effect of different treatments on the mortality of larvae incubated with (A): Seawater (control); (B): Methanol + seawater; (C): Untreated aflatoxins; (D): Toxins treated with $M$. arvensis leaf extract.

\section{Discussion}

Biodegradation of mycotoxins using fungi, bacteria and enzymes is now a known strategy to manage this issue [25-29]. However, not much data is available on the use of plant extracts for the same purpose. In our previous study, aqueous extracts of Acacia nilotica were used to detoxify aflatoxins in maize [30]. Whereas, this study was planned to explore the potential of a common medicinal herb, i.e., Mentha arvensis, to detoxify or inactivate aflatoxins in maize. In various trials, $M$. arvensis significantly degraded both AFB1 and AFB2 even when checked after three hours of incubation at the lowest tested temperature and $\mathrm{pH}$. However, the percentage of degradation increased with increases in incubation time, temperature and $\mathrm{pH}$.

The high temperature always supports chemical reactions. In in vitro assays, an increase in temperature significantly increased the percentage of degradation of aflatoxins. However, the overall results could be due to the synergistic effect of moisture and high temperature [31]. Many earlier workers have reported similar effects of high temperature on the rate of degradation of mycotoxins $[18,32,33]$. However, for further in vitro trials, $30^{\circ} \mathrm{C}$ was selected to eliminate any bad effect of high temperature on food chemistry. Moreover, $30{ }^{\circ} \mathrm{C}$ is quite close to the room temperature and prevailing temperature of storehouses in Pakistan during summer. This selection can make degradation a cost-effective approach at the industrial level.

After temperature, $\mathrm{pH}$ also plays an important role in governing a reaction speed. In this study, basic $\mathrm{pH}$ supported degradation reactions. Méndez-Albores, et al. [34] showed that a decrease in aflatoxins fluorescence due to a change in coumarin moiety in alkaline conditions. In the present investigation, the highest degradation was achieved at $\mathrm{pH} 10$. However, $\mathrm{pH} 8$ was selected for further trials to avoid the possibility of aflatoxins being unstable and sensitive at the basic $\mathrm{pH}$. As $\mathrm{pH} 8$ is 100 times less alkaline than $\mathrm{pH} 10$ and the percentage of biodegradation was also found comparable to $\mathrm{pH} 10, \mathrm{pH} 8$ was preferred for in vitro trials using detached maize stock. 
Aflatoxin B1 was converted into seven different compounds. A total of $28 \%$ of these compounds were formed due to the modification of the lactone ring. The lactone ring is responsible for the toxicity, carcinogenicity and fluorescence of aflatoxin B1 [35]. Modification in the lactone ring of AFB1 results in the loss of these properties, as observed in this study. Another $28 \%$ were formed due to the removal of the methoxy group from the side chain of benzene. A total of $14 \%$ of compounds were produced after the removal of the double bond in the furan ring. The presence of the double bond in the furan ring is another feature of AFB1 responsible for its toxicity and carcinogenic properties [36]. The rest of the compounds were formed by the loss of $\mathrm{H}_{2}$ and $\mathrm{CO}_{2}$.

Aflatoxin B2 was degraded to three different compounds by the replacement of the methoxy group with a hydroxyl group, the addition of $\mathrm{H}_{2}$ and removal of $\mathrm{CO}$ from the lactone ring and the side chain of the benzene ring. All these changes synergistically reduced the toxicity of the toxin significantly.

These biodegraded products were tested for biological toxicity against brine shrimps. Earlier studies have shown that the eggs and larvae of brine shrimps are susceptible to mycotoxins and hence have been used by several workers as a biological indicator of toxicity of mycotoxins in food and feed $[37,38]$. The percentage mortality of brine shrimps significantly decreased when they were incubated in water containing treated toxins. Hence it was confirmed that the treatment of selected toxins with aqueous extracts of $M$. arvensis can biodegrade targeted toxins into significantly less toxic compounds. Due to its efficacy, leaves of $M$. arvensis or its aqueous extract can be used as additives in food and feed for making bioactive packaging and mycotoxins binding commercial products to minimize the production and toxicity of aflatoxins. The identification of bioactive components from tested extracts and their use can increase the percentages of biodegradation.

\section{Materials and Methods}

\subsection{Preparation of Plant Extract}

Plants of Mentha arvensis were collected from Islamabad, Pakistan, in the month of May. Leaves and shoots of $M$. arvensis were first surface sterilized with $1 \%$ sodium hypochlorite for $10 \mathrm{~min}$, followed by several washings with sterile distilled water. A total of $10 \mathrm{~g}$ of plant material was homogenized with $10 \mathrm{~mL}$ sterilized distilled water to obtain the aqueous extract. This mixture was then filtered using a muslin cloth and centrifuged at 14,000 rpm for $20 \mathrm{~min}$. The supernatant obtained was sterilized by passing through a $0.2 \mu \mathrm{m}$ syringe filter assembly before using in further trials.

\subsection{In Vitro Toxin Inactivation Assay}

Firstly, the working solution was prepared consisting of methanol and water (60:40, $v / v)$. A total of $50 \mu \mathrm{L}$ of the working solution containing $50 \mu \mathrm{g} / \mathrm{L}$ AFB2 and $100 \mu \mathrm{g} / \mathrm{L}$ AFB1 mixed with $250 \mu \mathrm{L}$ of $M$. arvensis aqueous extract was incubated for various time intervals. After the incubation period, $500 \mu \mathrm{L}$ of chloroform was added to terminate the reaction. The mixture was thoroughly vortexed to extract the residual toxin. This was followed by the separation of the chloroform fraction at low-speed $(5000 \mathrm{rpm})$ centrifugation. The organic phase was then evaporated to dryness under a gentle stream of nitrogen and dissolved in $100 \mu \mathrm{L}$ of methanol. A total of $50 \mu \mathrm{L}$ of toxin in $250 \mu \mathrm{L}$ of water acted as the control that was also incubated under parallel conditions.

\subsection{Estimation of Optimal $\mathrm{pH}$ for In Vitro Biodegradation Using Plant Extracts}

The $\mathrm{pH}$ of the aqueous extracts was adjusted between 2.0 and 10.0 using $1 \mathrm{~N} \mathrm{HCl}$ or $1 \mathrm{~N}$ $\mathrm{NaOH}$ and then checked for their biodegradation potential. Distilled water and untreated extract acted as a control. 


\subsection{Estimating Optimal Temperature and Incubation Time for In Vitro Biodegradation Using Plant Extracts}

Plant extracts were incubated with toxins at $25,30,35,40,45,50,55$ and $60{ }^{\circ} \mathrm{C}$ for 3 , $6,12,24,48$ and $72 \mathrm{~h}$. The toxin content in various reaction mixtures was estimated using high-performance liquid chromatography.

\subsection{In Vitro Biodegradation of Toxins in Maize Samples Using Plant Extracts}

Maize stock samples containing mature grains were spiked with aflatoxins (B1 $100 \mu \mathrm{g} / \mathrm{L}$ and B2 $50 \mu \mathrm{g} / \mathrm{L})$. First, samples were decontaminated as described by Das and Mishra [39] with minor modifications. Briefly, $10 \mathrm{~g}$ of maize samples were kept in each $250 \mathrm{~mL}$ Erlenmeyer flask and spiked with $3 \mathrm{~mL}$ of aflatoxins (with concentration B1 $100 \mu \mathrm{g} \mathrm{L}{ }^{-1}$ and B2 $50 \mu \mathrm{g} \mathrm{L}^{-1}$ ). These samples were then incubated with $10 \mathrm{~mL}$ of aforementioned plant extracts at $30{ }^{\circ} \mathrm{C}$ for $72 \mathrm{~h}$. Afterward, aflatoxin was extracted according to the modified method of Stroka, et al. [40]. Maize samples were incubated with water:acetonitrile (15:85\%) in a shaking water bath for $2 \mathrm{~h}$. Afterward, the extracts were filtered through filter paper (Whatman, Inc., Clifton, NJ, USA). Afterward, the filtrate was passed through an afla immunoaffinity column in a solid-phase extraction assembly. The toxins were slowly eluted from the column with $1 \mathrm{~mL}$ of methanol in a glass vial, which was further analyzed by TLC and HPLC. The control comprised an untreated maize sample, a sample with plant extract without toxin and a sample with toxin without plant extract. Each experiment was performed in triplicate.

\subsection{Detection and Quantification of Treated Toxins}

TLC was used to detect the products of treated toxins. Chloroform and methanol fraction $(20 \mu \mathrm{L})$, of both treated and control toxins, were run on $0.25 \mathrm{~mm}$ silica gel (60F254 $20 \times 20 \mathrm{~cm}$, Merck, Darmstadt, Germany) TLC plates. These plates were then developed in 92:8 v/v chloroform:acetone and viewed under UV light (365 nm). Quantification of treated and untreated toxins after derivatization was performed through Agilent 1100 series HPLC (Agilent Technologies, Santa Clara, CA, USA) fitted with a reversed-phase C18 column (Merck, Darmstadt, Germany) and a fluorescence detector. Water:methanol:acetonitrile (60:20:20) was used as the mobile phase with a flow rate of $1 \mathrm{~mL} / \mathrm{min}$. Aflatoxins were detected at excitation and emission wavelengths of 360 and $440 \mathrm{~nm}$, respectively. Using a series of calibration solutions in methanol, calibration curves were drawn for HPLC method validation. Each standard solution was chromatographed in duplicate. The identification of degraded toxin metabolites was performed by mass spectral studies.

\subsection{LCMS Analysis of Bio-Degraded Toxins}

Analyses of the degraded toxins and their products were carried out by a surveyor LC system equipped with a mass spectrophotometer and PDA plus detectors (Thermo Fisher Scientific Waltham, MA, USA). All analyses were performed in triplicate using a luna phenomenex C18 column $(150 \times 4.6 \mathrm{~mm}, 3 \mu \mathrm{m})$, in isocratic mode. The column temperature was maintained at $30^{\circ} \mathrm{C}$. The injection volume was $10 \mu \mathrm{L}$, whereas the mobile phase consisted of methanol:acetonitrile:water in 22.5:22.5:55.0 $\mathrm{v} / \mathrm{v}$ ratio. The flow rate was $0.5 \mathrm{~mL} / \mathrm{min}$. The capillary temperature was $335^{\circ} \mathrm{C}$, sheath gas flow and auxiliary gas flow were 20 and $4 \mathrm{~L} / \mathrm{min}$, respectively. Source voltage, capillary voltage and tube lens voltage were $5 \mathrm{KV}, 49 \mathrm{~V}$ and $120 \mathrm{~V}$, respectively.

\subsection{ESI-MS/MS Conditions for Aflatoxins through Direct Insertion Pump}

Mass spectrometry/mass spectrometry was carried out using a Thermo Scientific LTQ XL System equipped with electrospray ionization (ESI) source operating in the positive ionization mode with a capillary voltage of $49.0 \mathrm{~V}$, a source voltage of $5.0 \mathrm{KV}$, a tube lens voltage of $110 \mathrm{~V}$ and a capillary temperature of $275^{\circ} \mathrm{C}$. The sheath and auxiliary gas flow were adjusted to 3 and $0.4 \mathrm{~L} / \mathrm{min}$, respectively, to get a stable spray. Data were collected in positive mode within the range of 100 to $500 \mathrm{~m} / \mathrm{z}$. The identification of 
unknown compounds was based on the accurate measurement of the mass of parent ions and fragments, as well as other useful MS/MS spectrum information.

\subsection{Determining Bio-Toxicity of Degraded Products}

The bio-toxicity of degraded toxin products was checked using a brine shrimp (Artemia salina) bioassay following the method developed by Solis 36, with some modifications. Brine shrimp eggs, 100-200 mg, were hatched in artificial seawater $(34 \mathrm{~g}$ sea salt/L of deionized water) by incubation at $26^{\circ} \mathrm{C}$ under a $60 \mathrm{~W}$ lamp. After separating from shells, the nauplii were transferred to fresh seawater. A total of $300 \mu \mathrm{L}$ of treated and untreated AFB1 (50, 100, 200, $300 \mu \mathrm{g} / \mathrm{L})$ and AFB2 $(20,50,90,130 \mu \mathrm{g} / \mathrm{L})$ was added to 96-well plates and dried overnight. After solvent evaporation, the toxins were redissolved in seawater $(200 \mu \mathrm{L})$. Another $200 \mu \mathrm{L}$ of seawater containing $40-45$ organisms was pipetted into each well, making a final volume of $400 \mu \mathrm{L}$ and were incubated at $26^{\circ} \mathrm{C}$ for $24-96 \mathrm{~h}$. Methanol was used as positive control, while for the negative control, seawater was used. Mortality was calculated by counting the immobile (dead) larvae under a stereomicroscope. The test was performed in triplicate.

\subsection{Statistical Analysis}

Five replicates were performed of each treatment, and the values are presented as mean \pm standard error. Data acquired in the above experiments were subjected to statistical analyses using DSSTAT software. Analysis of variance (ANOVA) and differences among the means was calculated for significance at $p \leq 0.05$ using Tukey's multiple range test.

Author Contributions: Conceptualization, T.A.; methodology, W.I. and M.I.; software, M.A. and W.A.; validation; formal analysis, W.I. and T.A.; investigation; resources, G.L.; data curation, W.I.; writing-original draft preparation, T.A.; writing-review and editing, T.A. and W.A.; supervision, T.A.; project administration, G.L.; funding acquisition, G.L. All authors have read and agreed to the published version of the manuscript.

Funding: This study was supported by the projects of Key-Area Research and Development Program of Guangdong Province (Project No: 2020B0202090002) and Guangdong Agriculture Department of China (Project No: 2021KJ122).

Institutional Review Board Statement: Not applicable.

Informed Consent Statement: Not applicable.

Data Availability Statement: Data is contained within the article.

Conflicts of Interest: The authors declare no conflict of interest.

\section{References}

1. Creppy, E.E. Update of survey, regulation and toxic effects of mycotoxins in Europe. Toxicol. Lett. 2002, 127, 19-28. [CrossRef]

2. Tabuc, C.; Marin, D.; Guerre, P.; Sesan, T.; Bailly, J.D. Molds and mycotoxin content of cereals in southeastern Romania. J. Food Prot. 2009, 72, 662-665. [CrossRef]

3. Ghiasian, S.A.; Shephard, G.S.; Yazdanpanah, H. Natural occurrence of aflatoxins from maize in Iran. Mycopathologia 2011, 172, 153-160. [CrossRef]

4. Khatoon, S.; Hanif, N.Q.; Tahira, I.; Sultana, N.; Sultana, K.; Ayub, N. Natural occurrence of aflatoxins, zearalenone and trichothecenes in maize grown in Pakistan. Pak. J. Bot. 2012, 44, 231-236.

5. Majeed, S.; Iqbal, M.; Asi, M.R.; Iqbal, S.Z. Aflatoxins and ochratoxin A contamination in rice, corn and corn products from Punjab, Pakistan. J. Cereal Sci. 2013, 58, 446-450. [CrossRef]

6. Munir, M.A.; Saleem, M.; Malik, Z.R.; Ahmed, M.; Ali, A. Incidence of aflatoxin contamination in non-perishable food commodities. J. Pak. Med. Assoc. 1989, 39, 154-157.

7. Shah, H.U.; Simpson, T.J.; Alam, S.; Khattak, K.F.; Perveen, S. Mould incidence and mycotoxin contamination in maize kernels from Swat Valley, North West Frontier Province of Pakistan. Food Chem. Toxicol. 2010, 48, 1111-1116. [CrossRef] [PubMed]

8. Iram, W.; Anjum, T.; Abbas, M.; Khan, A.M. Aflatoxins and ochratoxin A in maize of Punjab, Pakistan. Food Addit. Contam. Part B 2014, 7, 57-62. [CrossRef]

9. Luo, Y.; Liu, X.; Li, J. Updating techniques on controlling mycotoxins-A review. Food Control 2018, 89, 123-132. [CrossRef] 
10. Gowda, N.K.S.; Swamy, H.; Mahajan, P. Aflatoxins-Recent Advances and Future Prospects; Recent Advances for Control, Counteraction and Amelioration of Potential Aflatoxins in Animal Feeds; IntechOpen: London, UK, 2013; pp. 129-140.

11. Nazhand, A.; Durazzo, A.; Lucarini, M.; Souto, E.B.; Santini, A. Characteristics, occurrence, detection and detoxification of aflatoxins in foods and feeds. Foods 2020, 9, 644. [CrossRef]

12. Ciegler, A.; Lillehoj, E.B.; Peterson, R.E.; Hall, H.H. Microbial detoxification of aflatoxin. Appl. Microbiol. 1966, 14, 934-939. [CrossRef] [PubMed]

13. Minuye, M. Aflatoxin Reduction Mechanism of Probiotics. J. Prob. Health 2021, 9, 235.

14. Al-Rahmah, N.; Mostafa, A.; Abdel-Megeed, A. Antifungal and antiaflatoxigenic activities of some plant extracts. Afr. J. Microbiol. Res. 2011, 5, 1342-1348. [CrossRef]

15. El-Nagerabi, S.A.F.; Al-Bahry, S.N.; Elshafie, A.E.; AlHilali, S. Effect of Hibiscus sabdariffa extract and Nigella sativa oil on the growth and aflatoxin B1 production of Aspergillus flavus and Aspergillus parasiticus strains. Food Control 2012, 25, 59-63. [CrossRef]

16. Hamad, G.M.; Mohdaly, A.A.A.; El-Nogoumy, B.A.; Ramadan, M.F.; Hassan, S.A.; Zeitoun, A.M. Detoxification of Aflatoxin B1 and Ochratoxin A Using Salvia farinacea and Azadirachta indica Water Extract and Application in Meat Products. Appl. Biochem. Biotechnol. 2021, 193, 3098-3120. [CrossRef] [PubMed]

17. Shanakhat, H.; Sorrentino, A.; Raiola, A.; Romano, A.; Masi, P.; Cavella, S. Current methods for mycotoxins analysis and innovative strategies for their reduction in cereals: An overview. J. Sci. Food Agric. 2018, 98, 4003-4013. [CrossRef]

18. Velazhahan, R.; Vijayanandraj, S.; Vijayasamundeeswari, A.; Paranidharan, V.; Samiyappan, R.; Iwamoto, T.; Friebe, B.; Muthukrishnan, S. Detoxification of aflatoxins by seed extracts of the medicinal plant, Trachyspermum ammi (L.) Sprague ex Turrill-structural analysis and biological toxicity of degradation product of aflatoxin G1. Food Control. 2010, 21, 719-725. [CrossRef]

19. Rajan, M.; Gupta, P.; Kumar, A. Promising Antiviral Molecules from Ayurvedic Herbs and Spices against COVID-19. Chin. J. Integr. Med. 2021, 27, 243-244. [CrossRef]

20. Ghani, A. Medicinal Plants of Bangladesh: Chemical Constituents and Uses; Asiatic Society of Bangladesh: Dhaka, Bangladesh, 1998.

21. Satyavati, G.V.; Raina, M.K.; Sharma, M. Medicinal Plants of India; Indian Council of Medical Research: New Delhi, India, 1987; Volume 2.

22. Verma, R.S.; Rahman, L.; Verma, R.K.; Chauhan, A.; Yadav, A.K.; Singh, A. Essential oil composition of menthol mint (Mentha arvensis) and peppermint (Mentha piperita) cultivars at different stages of plant growth from Kumaon region of Western Himalaya. Open Access J. Med. Aromat. Plants 2010, 1, 13.

23. Iram, W.; Anjum, T.; Jabeen, R.; Abbas, M. Isolation of stored maize mycoflora, identification of aflatoxigenic fungi and its inhibition using medicinal plant extracts. Int. J. Agric. Biol. 2018, 20, 2149-2160.

24. Koka, J.A.; Wani, A.H.; Bhat, M.Y.; Parveen, S. Antifungal activity of ethanolic and aqueous leaf extracts of Taraxicum officinale and Mentha arvensis on the growth of some selected fungal species under in vitro conditions. Int. J. Pure Appl. Biosci. 2017, 5, 1170-1176. [CrossRef]

25. Afshar, P.; Shokrzadeh, M.; Raeisi, S.N.; Ghorbani-HasanSaraei, A.; Nasiraii, L.R. Aflatoxins biodetoxification strategies based on probiotic bacteria. Toxicon 2020, 178, 50-58. [CrossRef] [PubMed]

26. Guo, Y.; Zhang, Y.; Wei, C.; Ma, Q.; Ji, C.; Zhang, J.; Zhao, L. Efficacy of Bacillus subtilis ANSB060 biodegradation product for the reduction of the milk aflatoxin M1 content of dairy cows exposed to aflatoxin B1. Toxins 2019, 11, 161. [CrossRef]

27. Hassan, Y.I.; Zhou, T. Promising detoxification strategies to mitigate mycotoxins in food and feed. Toxins 2018, 10, 116. [CrossRef]

28. Ji, C.; Fan, Y.; Zhao, L. Review on biological degradation of mycotoxins. Anim. Nutr. 2016, 2, 127-133. [CrossRef]

29. Yang, X.; Chen, X.; Song, Z.; Zhang, X.; Zhang, J.; Mei, S. Antifungal, plant growth-promoting, and mycotoxin detoxication activities of Burkholderia sp. strain XHY-12. 3 Biotech 2020, 10, 158. [CrossRef] [PubMed]

30. Anjum, T.; Iram, W.; Iqbal, M.; Ghaffar, A.; Abbas, M. Identification of degradation products of aflatoxin B1 and B2 resulting after their biodetoxification by aqueous extracts of Acacia nilotica. World Mycotoxin J. 2020, 13, 499-514. [CrossRef]

31. Rustom, I.Y.S. Aflatoxin in food and feed: Occurrence, legislation and inactivation by physical methods. Food Chem. 1997, 59, 57-67. [CrossRef]

32. Hajare, S.S.; Hajare, S.N.; Sharma, A. Aflatoxin inactivation using aqueous extract of ajowan (Trachyspermum ammi) seeds. J. Food Sci. 2005, 70, C29-C34. [CrossRef]

33. Vijayanandraj, S.; Brinda, R.; Kannan, K.; Adhithya, R.; Vinothini, S.; Senthil, K.; Chinta, R.R.; Paranidharan, V.; Velazhahan, R. Detoxification of aflatoxin B1 by an aqueous extract from leaves of Adhatoda vasica Nees. Microbiol. Res. 2014, 169, 294-300. [CrossRef]

34. Méndez-Albores, J.A.; Villa, G.A.; Del Rio-García, J.C.; Martínez, E.M. Aflatoxin-detoxification achieved with Mexican traditional nixtamalization process (MTNP) is reversible. J. Sci. Food Agric. 2004, 84, 1611-1614. [CrossRef]

35. Jackson, L.W.; Pryor, B.M. Degradation of aflatoxin B 1 from naturally contaminated maize using the edible fungus Pleurotus ostreatus. AMB Express 2017, 7, 110. [CrossRef] [PubMed]

36. Wang, F.; Xie, F.; Xue, X.; Wang, Z.; Fan, B.; Ha, Y. Structure elucidation and toxicity analyses of the radiolytic products of aflatoxin B1 in methanol-water solution. J. Hazard. Mater. 2011, 192, 1192-1202. [CrossRef] [PubMed]

37. Durakovic, S.; Delas, F.; Durakovic, L. Microbial indicators of safety and quality of foods. In Modern Microbiology of Food; Durakovic, S., Ed.; Kugler: London, UK, 2002; pp. 249-282. 
38. Moretti, A.; Mule, G.; Ritieni, A.; Logrieco, A. Further data on the production of beauvericin, enniatins and fusaproliferin and toxicity to Artemia salina by Fusarium species of Gibberella fujikuroi species complex. Int. J. Food Microbiol. 2007, 118, 158-163. [CrossRef]

39. Das, C.; Mishra, H. In vitro Degradation of Aflatoxin B1in Groundnut (Arachis hypogea) Meal by Horse Radish Peroxidase. LWT-Food Sci. Technol. 2000, 33, 308-312. [CrossRef]

40. Stroka, J.; Van Otterdijk, R.; Anklam, E. Immunoaffinity column clean-up prior to thin-layer chromatography for the determination of aflatoxins in various food matrices. J. Chromatogr. A 2000, 904, 251-256. [CrossRef] 